\title{
FAUNA DE ESCARABAJOS MELOLÓNTIDOS (COLEOPTERA: SCARABAEOIDEA) EN EL MUNICIPIO DE VILLAFLORES, CHIAPAS, MÉXICO
}

\author{
Cutberto Pacheco Floresi', Adriana E. Castro Ramírez', \\ Miguel A. Morón², Benigno GóMEZ Y GóMEz1 \\ ${ }^{1}$ El Colegio de la Frontera Sur -Unidad San Cristóbal de Las Casas, Chiapas, A.P. 63 San \\ Cristóbal de Las Casas, Chiapas 29290 MÉXICO. \\ cpacheco35@hotmail.com, acastro@sclc.ecosur.mx \\ 2Departamento de Biología de Suelos, Instituto de Ecología, A.C. A. P. 63 \\ Xalapa, Veracruz, 91000 MÉXICO. \\ miguel.moron@inecol.edu.mx
}

\begin{abstract}
RESUMEN
Como un primer acercamiento al conocimiento de los escarabajos melolóntidos de la región Frailesca de Chiapas, México, caracterizada por un ambiente original de bosque tropical caducifolio, en su mayor parte ahora reemplazado por grandes extensiones con producción agrícola y ganadera, se realizó la presente investigación en los ejidos de Francisco Villa, Cuauhtémoc, El Jardín y la ciudad de Villaflores, pertenecientes al municipio de Villaflores. En parcelas agrícolas se hicieron recolectas sistemáticas, diurnas y nocturnas, de escarabajos adultos durante los meses de mayo a septiembre de 2006. Se obtuvieron 6,780 ejemplares, que representan a cuatro subfamilias, ocho tribus, 17 géneros y 46 especies. Los géneros con mayor número de especies e individuos fueron Phyllophaga, Diplotaxis y Ligyrus; junto con los escarabajos de los géneros Cyclocephala, Anomala y Strigoderma cubren el 88.7 $\%$ de la abundancia total. Se incluye una lista actualizada de las 368 especies de Melolonthidae registradas en el estado de Chiapas.
\end{abstract}

Palabras clave: Phyllophaga, Diplotaxis, Ligyrus, región Frailesca, bosque tropical caducifolio, hospederos, maíz.

\begin{abstract}
A first approach to the knowledge of the Melolonthid beetles of the region Frailesca in the State of Chiapas, Mexico, is presented. Most part of the original tropical deciduous forest today is replaced by great extensions with agricultural and cattle production. Studied localities were Francisco Villa, Cuauhtémoc, El Jardín and the surrounds of Villaflores city, all from the municipality of Villaflores. Diurnal and nocturnal systematic collections of adult beetles were made in agricultural parcels during May to September, 2006, by mean of light traps, transects, and fruit traps We obtained 6,780 specimens that represent four subfamilies, eight tribes, 17 genera and 46 species. The genera with greatest number of species and individuals were Phyllophaga, Diplotaxis and Ligyrus; along with the species of the genera Cyclocephala, Anomala and Strigoderma cover
\end{abstract}


$88,7 \%$ of the total abundance. An updated list of the 368 species of Melolonthidae recorded in the state of Chiapas is included.

Key words: Phyllophaga, Diplotaxis, Ligyrus, Frailesca region, tropical deciduous forest, hosts, maize.

\section{INTRODUCCIÓN}

En el estado de Chiapas se han desarrollado algunos estudios faunísticos sobre escarabajos en áreas conservadas, pudiéndose citar el trabajo de Morón y colaboradores (1985) en Boca de Chajul, el de Gómez y Gómez (1998) en El Ocote, Morón-Ríos y Morón (2001) en la reserva de la Biosfera El Triunfo, el de PalaciosRíos y colaboradores (1990) en Yaxchilán, Morales-Morales et al. (2005) en Laguna Bélgica en Ocozocuautla. Mientras que en áreas agrícolas se tienen los estudios de Alcázar-Ruiz y colaboradores (2003) en Villa Las Rosas, y el de Castro-Ramírez y colaboradores (2005) en Los Altos de Chiapas; éste último considera los melolóntidos asociados a parcelas agrícolas tanto en estado larvario (plaga agrícola) como en adulto.

Sin embargo, no se han realizado estudios en el trópico seco, como la región Frailesca, caracterizada por un ambiente de bosque tropical caducifolio (Rzedowski 1978) y considerada la principal región productora de granos básicos en el estado; dicha vocación agropecuaria ha permitido grandes extensiones de monocultivo de maíz, con el uso indiscriminado de agroquímicos (SEMARNAP 1999), lo que posiblemente ha modificado la composición de la coleopterofauna cercana a las zonas de cultivos (Morón et al. 1998). Por ello como un primer acercamiento al conocimiento de la coleopterofauna en la región Frailesca, la presente investigación se propuso registrar las especies de escarabajos melolóntidos que habitan en el área agrícola de cuatro sitios en el municipio de Villa Flores.

\section{ÁREA DE ESTUDIO}

La investigación se realizó en los ejidos Francisco Villa, El Jardín, Cuauhtémoc y los alrededores de la ciudad de Villaflores (Fig.1). El municipio se ubica a $620 \mathrm{~m}$ de altitud, entre los $16^{\circ} 35^{\prime}$ y $16^{\circ} 10^{\prime}$ de latitud norte, y $93^{\circ} 03^{\prime}$ y $93^{\circ} 46^{\prime}$ de longitud oeste; predomina el clima cálido subhúmedo con lluvias en verano (Aw). La cabecera municipal tiene una temperatura media anual de $24.3^{\circ} \mathrm{C}$ y $1,209 \mathrm{~mm}$ de precipitación pluvial al año. La agricultura es una de las actividades más importantes en todo el municipio, en mayor escala se producen granos básicos como maíz (Zea mays L.), frijol (Phaseolus vulgaris L.), sorgo (Sorghum bicolor L.) y cacahuate (Arachis hypogaea L.) (Córdova 2002, Orozco 2002). 


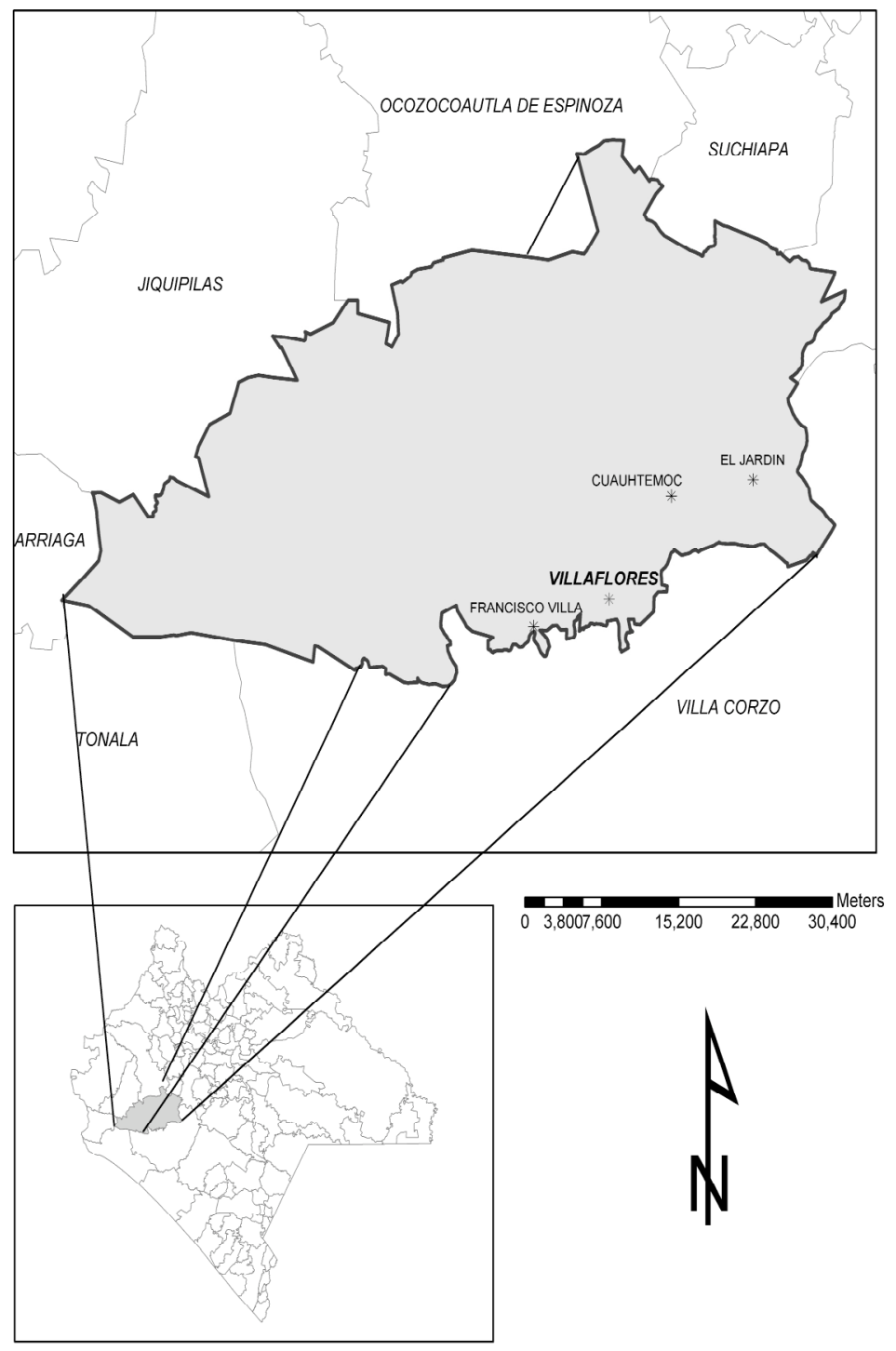

Figura 1

Localización de los sitios de estudio en el municipio de Villaflores, Chiapas, México (LAIGE-ECOSUR 2006). 


\section{MATERIAL Y MÉTODOS}

Durante los meses de mayo a septiembre de 2006 se realizaron recolectas sistemáticas, tres veces a la semana (lunes, miércoles y viernes), diurnas y nocturnas, de escarabajos adultos en una parcela agrícola de cada localidad. Las capturas se hicieron manualmente buscando los escarabajos sobre las diferentes plantas hospederas; en las recolectas nocturnas, de 19:00 a 21:30 hrs (horario de verano), se utilizó una trampa de luz de tipo pantalla (lámpara fluorescente de luz blanca 20 watts), se buscaron en alumbrado público y por medio de lampareo. En las recolectas diurnas se revisaron las plantas herbáceas y arbustivas presentes en transectos de $500 \mathrm{~m}$, en horario de 9:00 a 17:00 hrs. Además, en cada sitio se instalaron diez trampas con frutas fermentadas (piña o plátano) colocadas en árboles, la mitad de ellas ubicadas a $3 \mathrm{~m}$ y la otra mitad a $5 \mathrm{~m}$ de altura (sensu Morón 1995a, 1997). Los escarabajos se conservaron en alcohol isopropílico al 70\%, debidamente etiquetados. Se corroboraron las determinaciones en el Departamento de Biología de Suelos del Instituto de Ecología A. C., en Xalapa Veracruz; y en El Colegio de la Frontera Sur -Unidad San Cristóbal de Las Casas (ECOSUR-SC), Chiapas. Con la finalidad de obtener datos diarios sobre la precipitación de la zona de estudio, se instaló un pluviómetro (METRIC ${ }^{\circledR}$ ) en Villaflores. El material entomológico se encuentra depositado en la colección del proyecto Diversidad en Sistemas de Cultivos de ECOSUR-SC; con duplicados en las colecciones de ECOSUR-SC (ECOSC-E), ECOSUR-Unidad Tapachula, Chiapas y del Instituto de Ecología A. C. en Xalapa, Veracruz.

Para evaluar la importancia ecológica de los géneros registrados en la zona de Villaflores se les ubicó en los grupos funcionales propuestos por Morón y Deloya (1991) y Deloya (2006). Se realizó la revisión de literatura para actualizar el registro de las especies de escarabajos melolóntidos citados para el estado de Chiapas y su distribución local.

\section{RESULTADOS}

La emergencia de los escarabajos melolóntidos nocturnos se realizó entre las 20:10 y 20:30 hrs en promedio. Donde primero se dio fue en Villaflores, el dos de mayo, con una precipitación de tan sólo 3 mm (Fig. 2). En Francisco Villa (Fig. 3) y El Jardín (Fig. 4) la emergencia de melolóntidos inició el 22 de mayo; para el primer sitio se dio posterior a una precipitación acumulada de $13.6 \mathrm{~mm}$, y la mayor abundancia se obtuvo después de que llovieran $38 \mathrm{~mm}$ el 8 de junio. En El Jardín se obtuvo la mayor cantidad de escarabajos al inicio del periodo de lluvias. En la localidad de Cuauhtémoc se tuvo la emergencia más tardía, comenzó a inicios del mes de junio (2 de junio) (Fig. 5). 


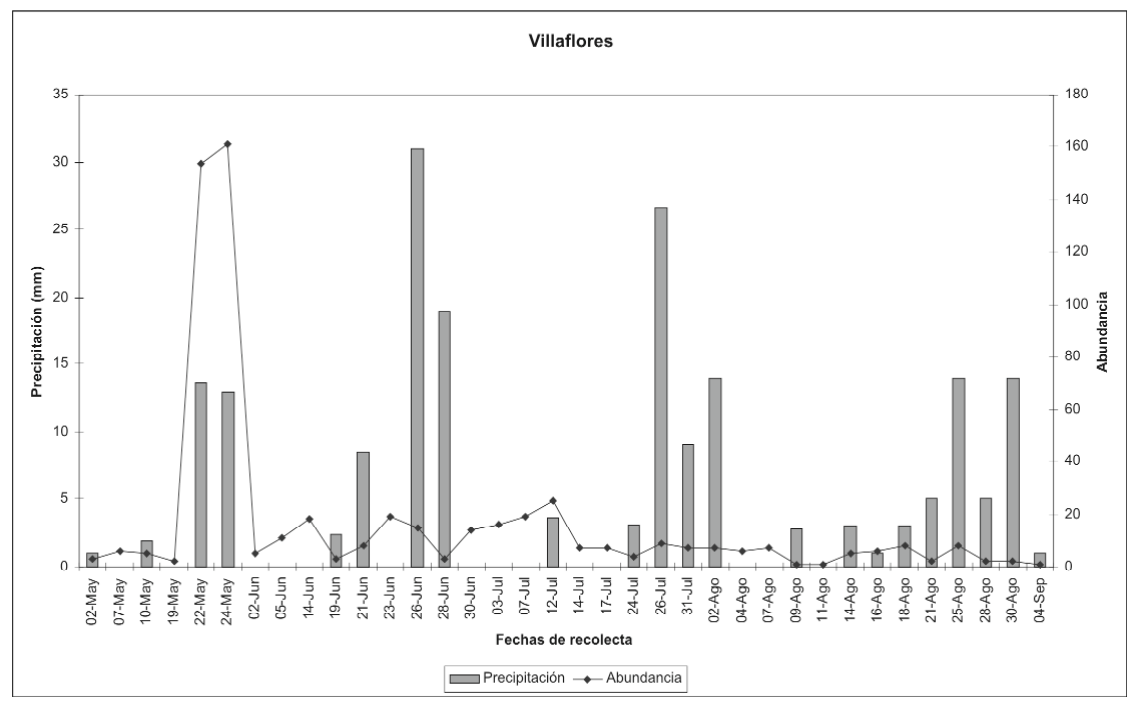

Figura 2

Abundancia de melolóntidos adultos, por fecha de recolecta, en relación con la precipitación en Villaflores, Chiapas (2006).

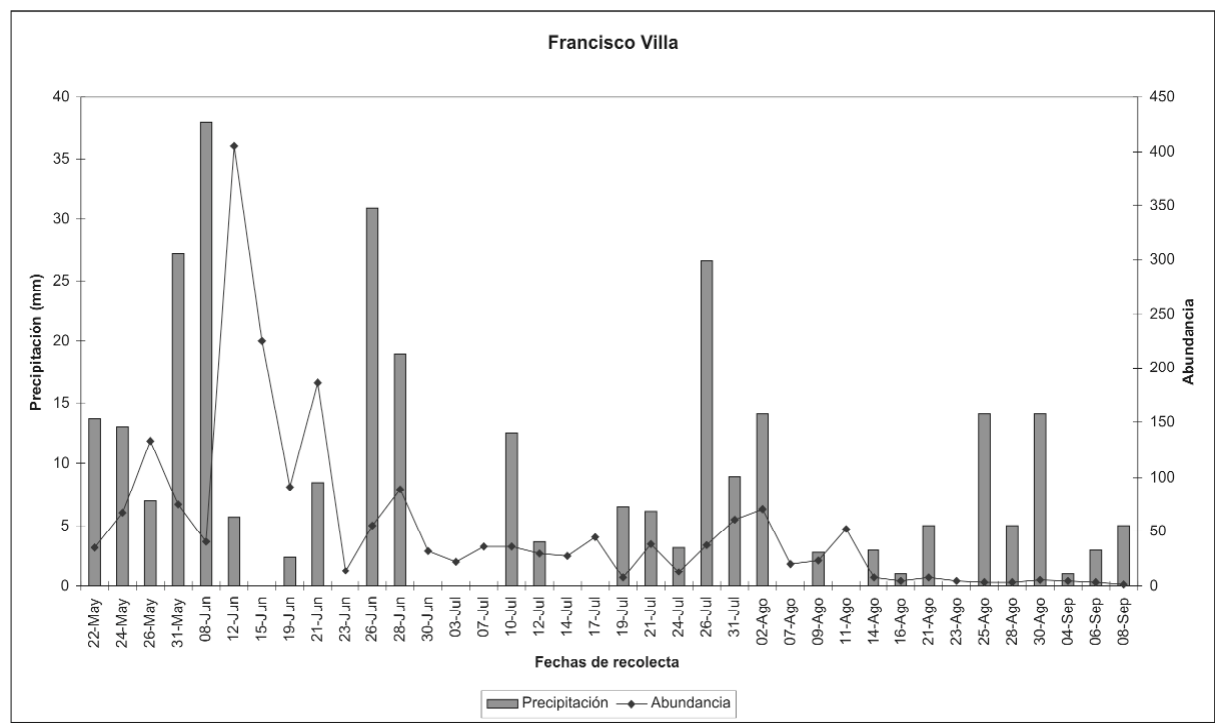

Figura 3

Abundancia de melolóntidos adultos, por fecha de recolecta, en relación con la precipitación en Francisco Villa, Chiapas (2006). 
Pacheco Flores et al.: Fauna de escarabajos melolóntidos (Coleoptera: Scarabeoidea) en Chiapas, México

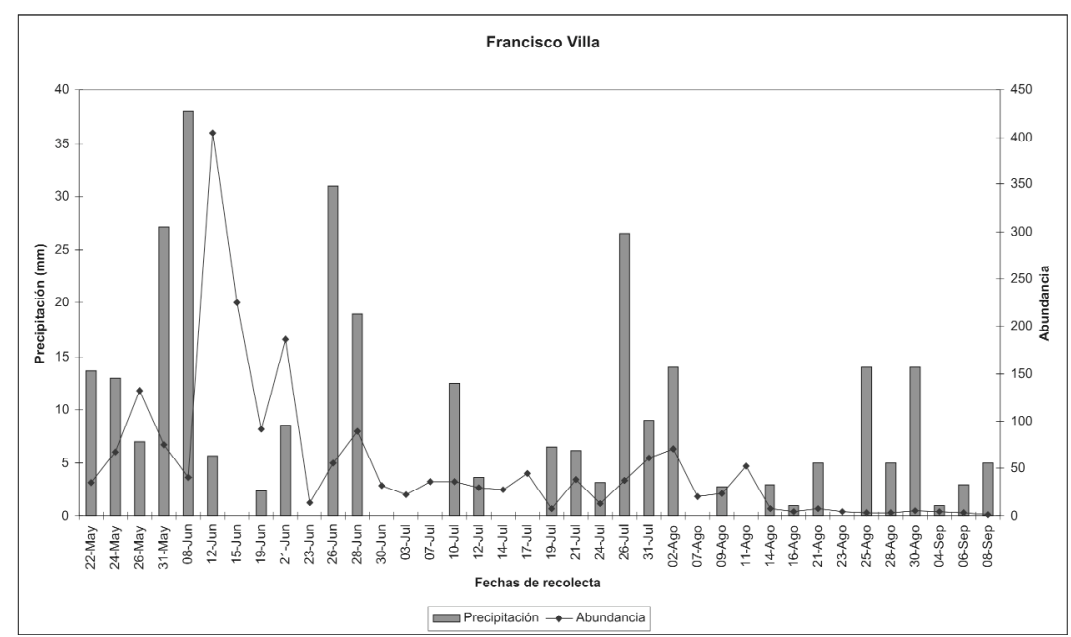

Figura 4

Abundancia de melolóntidos adultos por fecha de recolecta en relación con la precipitación en El Jardín, Chiapas (2006).

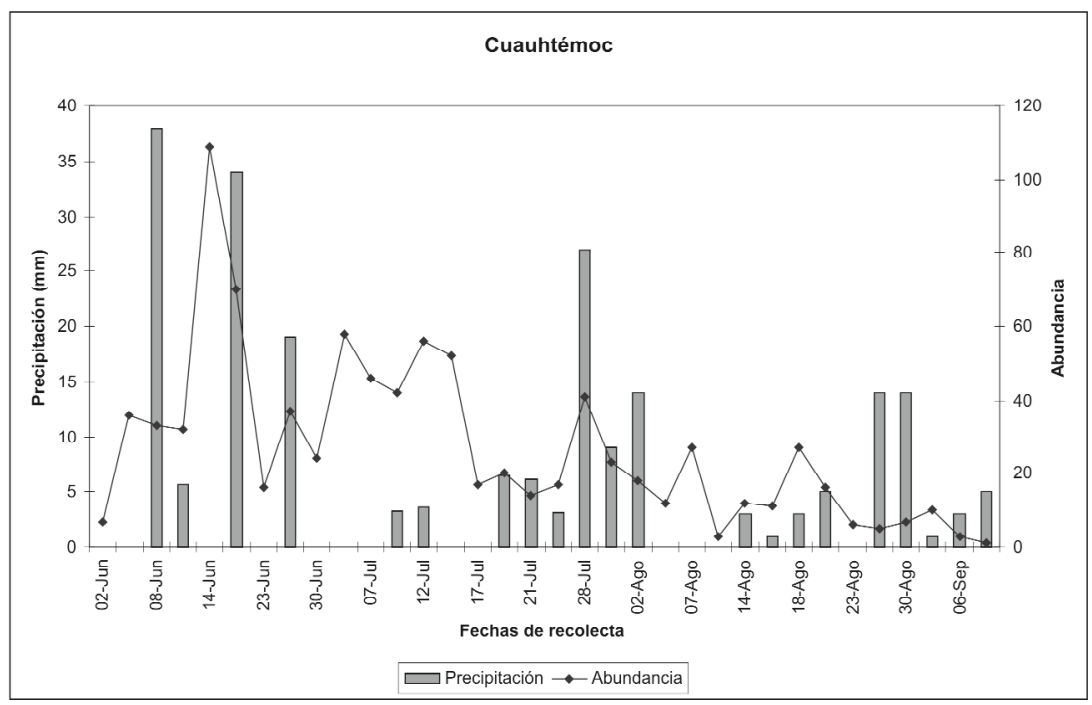

Figura 5

Abundancia de melolóntidos adultos, por fecha de recolecta, en relación con la precipitación en Cuauhtémoc, Chiapas (2006). 
En los cuatro sitios de estudio se recolectaron en total 6,780 ejemplares de Coleoptera Melolonthidae, que representan a cuatro subfamilias, ocho tribus, 17 géneros con 46 especies, en 152 eventos de captura (38/sitio) durante poco más de tres meses (fines de mayo-inicios de septiembre). A continuación se indica la composición específica para cada género.

\section{CETONIINAE}

Cotinis Burmeister, 1842. De este género se capturó la especie Cotinis mutabilis (Gory \& Percheron, 1833), en la trampa de fruta en El Jardín.

Euphoria Burmeister, 1842. Se obtuvieron dos especies: Euphoria pulchella (Gory \& Percheron, 1833) en las flores de Jatropha curcas L. (Euphorbiaceae) en El Jardín; y cinco ejemplares de Euphoria leucographa (Gory \& Percheron, 1833) fueron atraídos por la trampa de frutas en Francisco Villa.

\section{DYNASTINAE, Cyclocephalini}

Cyclocephala Latreille, 1829. De este género se recolectaron seis especies. Las más abundantes fueron Cyclocephala amazona (Linné, 1767) con 47 ejemplares en los cuatro sitios, y Cyclocephala lunulata Burmeister, 1847 con 28 individuos (Cuadro 1).

Dyscinetus Harold, 1869. De Dyscinetus laevipunctatus Bates, 1888 se recolectaron 53 individuos en Francisco Villa, seis en Cuauhtémoc y 24 en El Jardín, atraídos por los alumbrados públicos y las trampas de luz.

\section{Cuadro 1}

Número de individuos del género Cyclocephala, por especie y sitio de captura en el municipio de Villaflores, Chiapas.

\begin{tabular}{|c|c|c|c|c|}
\hline Especies & Fco. Villa & Villaflores & Cuauhtémoc & El Jardín \\
\hline Cyclocephala amazona (Linné, 1767) & 21 & 19 & 2 & 5 \\
\hline C. guttata Bates, 1888 & & 2 & & 2 \\
\hline C. lunulata Burmeister, 1847 & 12 & 3 & 3 & 10 \\
\hline C. mafaffa Burmeister, 1847 & 6 & & & \\
\hline C. melanocephala (Fabricius, 1775) & & & & 2 \\
\hline C. ovulum (Bates, 1888) & & & & 1 \\
\hline
\end{tabular}

\section{DYNASTINAE, Dynastini}

Golofa Hope, 1837. Se capturó un ejemplar de la especie Golofa tersander Burmeister, 1847, en Francisco Villa, en el alumbrado público. 


\section{DYNASTINAE, Oryctini}

Enema Hope, 1837. De Enema endymion Chevrolat, 1843 se recolectaron 18 ejemplares en El Jardín, cuatro en Villaflores, tres en Cuauhtémoc y uno en Francisco Villa; todos atraídos por las trampas de luz y alumbrados públicos.

Strategus Hope, 1837. Se recolectaron dos especies: Strategus aloeus Linné, 1758, ocho individuos en Francisco Villa, 13 en Villaflores, 19 en Cuauhtémoc y 13 en El Jardín, atraídos en los alumbrados públicos y trampa de luz; y Strategus jugurtha Burmeister, 1847, dos ejemplares en el alumbrado público de Francisco Villa.

Xyloryctes Hope, 1837. Se obtuvieron dos especies. Un ejemplar de Xyloryctes teuthras Bates, 1888 en Francisco Villa, atraído por la trampa de luz; y dos individuos de Xyloryctes lobicollis Bates, 1888, en la trampa de luz de El Jardín.

\section{DYNASTINAE, Pentodontini}

Euetheola Bates, 1888. Se recolectaron 188 ejemplares en Francisco Villa, 19 en Villaflores, 95 en Cuauhtémoc y 80 en El Jardín de la especie Euetheola bidentata Burmeister, 1847, atraídos por alumbrados públicos y trampas de luz.

Ligynus Burmeister, 1847. Se recolectaron tres especies. Ligyrus laevicollis Bates, 1888, se capturó un ejemplar en Villaflores en la trampa de luz. Ligyns nasutus (Burmeister, 1847) fue la especie más abundante del género; se recolectaron 474 ejemplares en Francisco Villa, 187 en Villaflores, 469 en Cuauhtémoc y 838 en El Jardín; atraídos en la trampa de luz y alumbrados públicos. Mientras que de Ligyrus sallei Bates, 1888 se capturaron tres ejemplares en Francisco Villa, uno en Villaflores y un ejemplar en El Jardín, en las trampas de luz.

\section{DYNASTINAE, Phileurini}

Phileurus Latreille, 1807. Se encontraron dos especies. Phileurus didymus (Linné, 1758), se obtuvo un ejemplar en El Jardín, en la trampa de luz; y de Phileurus valgus (Olivier, 1789) se recolectaron dos ejemplares en Francisco Villa, atraídos por la trampa de luz.

\section{RUTELINAE, Anomalini}

Anomala Samouelle, 1819. Se recolectaron cinco especies (Cuadro 2). La de mayor abundancia en tres de los sitios fue Anomala sticticoptera Blanchard, 1850, seguida por Anomala incostans Burmeister, 1847.

Callistethus Blanchard, 1850. Se capturaron en las trampas de luz 121 ejemplares de Callistethus bimaculatus Blanchard, 1850 en los cuatro sitios; 21 en Francisco Villa, cuatro en Villaflores, 41 en Cuauhtémoc y 55 en El Jardín.

Strigoderma Burmeister, 1844. Un ejemplar de Strigoderma sulcipennis Burmeister, 1844, se encontró en flores de Phaseolus vulgaris L. (Leguminosae), y siete ejemplares de Strigoderma mexicana Blanchard, 1850 fueron capturados en las flores de Amaranthus hybridus L. (Amaranthaceae) y Jatropha curcas L. (Euphorbiaceae) en Villaflores. En Francisco Villa fue posible capturar un ejemplar de Strigoderma aterrima Casey, 1915 en la flor de Mikania micrantha H.B.K. (Compositae), que representa el primer registro de esta especie para el estado de Chiapas.

Pelidnota Mac Leay, 1819. Se recolectaron tres especies, la de mayor abundancia fue Pelidnota punctulata Bates, 1888 con 27 individuos en Francisco Villa, dos en Cuauhtémoc 
y nueve en El Jardín, con trampa de luz; de $P$. aurescens Bates, 1888 se obtuvo un ejemplar en Cuauhtémoc; mientras que de Pelidnota strigosa Laporte, 1840 se capturaron 14 individuos en Francisco Villa, uno en Cuauhtémoc y 14 en El Jardín, en la trampa de luz.

\section{Cuadro 2}

Número de escarabajos del género Anomala, por especie y sitio de recolecta en el municipio de Villaflores, Chiapas.

\begin{tabular}{lcccc}
\hline Especies & Fco. Villa & Villaflores & Cuauhtémoc & El Jardín \\
\hline Anomala discoidalis Bates, 1888 & 2 & & 4 & 31 \\
A. histrionella Bates, 1888 & 4 & 1 & 1 & 3 \\
A. inconstans Burmeister, 1847 & 1 & 7 & 13 & 40 \\
A. sticticoptera Blanchard, 1850 & 6 & 3 & 1 & \\
Anomala s p & & & & \\
\hline
\end{tabular}

\section{MELOLONTHINAE, Melolonthini}

Phyllophaga Harris, 1827 se registraron siete especies (Cuadro 3). Las más abundantes fueron Phyllophaga lenis (Horn, 1887) con 1,190 individuos y Phyllophaga ravida (Blanchard, 1850) con 690 ejemplares, atraídos por los alumbrados públicos y trampas de luz en los cuatro sitios.

\section{Cuadro 3}

Número de escarabajos del género Phyllophaga, por especie y sitio de captura en el municipio de Villaflores, Chiapas.

\begin{tabular}{lcccc}
\hline Especies & Fco. Villa & Villaflores & Cuauhtémoc & El Jardín \\
\hline Phyllophaga chiapensis (Chapin, 1935) & 2 & 1 & 5 & 18 \\
Phyllophaga disca Saylor 1943 & 7 & & 8 & 152 \\
Phyllophaga fulviventris Moser, 1918 & 6 & & 1 & 16 \\
Phyllophaga lenis (Horn, 1887) & 89 & 213 & 86 & 802 \\
Phyllophaga multipora (Bates, 1888) & 49 & 19 & 1 & 1 \\
Phyllophaga ravida (Blanchard, 1850) & 169 & 22 & 44 & 455 \\
Phyllophaga sp & & & 1 & \\
\hline
\end{tabular}


Diplotaxis Kirby, 1837. Se capturaron cinco especies en los cuatro sitios (Cuadro 4). Siendo Diplotaxis aff. megapleura la más abundante con 1,553 ejemplares recolectados en las trampas de luz y de frutas, así como en el follaje de Acacia pennatula (S. \& C.) Benth. (Leguminosae); Mimosa albida H. \& B. Willd. (Leguminosae), Guazuma ulmifolia Lam. (Sterculiaceae), Waltheria americana L. (Sterculiaceae), Scoparia dulces L. (Scrophulariaceae) y Sida rhombifolia L. (Malvaceae). Los pocos individuos capturados de las especies Diplotaxis aff. denigrata y D. aff. puncticollis fueron atraídos por la trampa de luz. Los cien ejemplares de $D$. hallei atraídos por las luces en las cuatro localidades representan el primer registro de esta especie para el estado de Chiapas.

\section{Cuadro 4}

Número de escarabajos del género Diplotaxis, por especie y sitio de recolecta en el municipio de Villaflores, Chiapas.

\begin{tabular}{lcccc}
\hline Especies & Fco. Villa & Villaflores & Cuauhtémoc & El Jardín \\
\hline Diplotaxis aff. denigrata Bates, 1889 & 32 & 3 & 9 & 2 \\
D. aff. hallei Vaurie, 1958 & 804 & 49 & 91 & 609 \\
D. aff. megapleura Vaurie, 1960 & 1 & & 1 & 4 \\
D. aff. puberea Bates, 1887 & 2 & & \\
D. aff. puncticollis Moser, 1918 & & & \\
\hline
\end{tabular}

\section{Hábitos alimentarios de los Melolonthidae en Villaflores.}

De acuerdo con los criterios de Morón y Deloya (1991) y Deloya (2006) las especies de coleópteros Melolonthidae recolectados en el municipio de Villaflores pueden incluirse en tres grupos funcionales: sapro-fitófagos, fitófagos y depredadores (Cuadro 5). De éstos datos se deduce que la mayoría de las especies de melolóntidos presentes en las parcelas agrícolas estudiadas tienen larvas rizófagas y adultos fitófagos.

\section{Lista de melolóntidos citados en Chiapas.}

Con base en la revisión actualizada de literatura se encontró que la familia Melolonthidae del estado de Chiapas está representada por 366 especies de cinco subfamilias (Melolonthinae, Cetoniinae, Trichiinae, Dynastinae y Rutelinae) (Anexo 1). En este trabajo se incluyen dos especies que no han sido citadas para Chiapas, lo que modifica ligeramente el inventario a 368 especies de melolóntidos en el estado. 


\section{Cuadro 5}

Gremios alimentarios de melolóntidos encontrados en el municipio de Villaflores, Chiapas.

\begin{tabular}{|l|l|l|c|}
\hline Grupo funcional & Gremio (larva-adulto) & \multicolumn{1}{|c}{ Géneros } & $\%$ de especies \\
\hline \multirow{5}{*}{ Sapro-fitófago } & Sapro-melífagos & $\begin{array}{l}\text { Cotinis, Cyclocephala } \\
\text { Euphoria, Dyscinetus }\end{array}$ & 21.7 \\
\cline { 2 - 4 } & Xilo-filófago & Pelidnota & 6.5 \\
\cline { 2 - 4 } & Sapro-antófagos & Strigoderma & 6.5 \\
\cline { 2 - 4 } & Sapro-caulófagos & $\begin{array}{l}\text { Ligyrus, Strategus, Golofa, } \\
\text { Xyloryctes, Euetheola, Enema }\end{array}$ & 19.5 \\
\hline Fitófagos & Rizo-filófago & $\begin{array}{l}\text { Phyllophaga, Diplotaxis, } \\
\text { Anomala, Callistethus }\end{array}$ & 39.1 \\
\hline depredador & Depredador & Phileurus & 6.5 \\
\hline
\end{tabular}

\section{DISCUSIÓN}

Al comparar la riqueza de especies obtenida en el municipio de Villaflores (46 especies) con otros estudios realizados en el estado de Chiapas, se observa que es ligeramente menor a la encontrada en Villa Las Rosas (50 especies) (Alcázar-Ruiz et al. 2003) y de Laguna Bélgica en el municipio de Ocozocuautla (56 especies) (Morales-Morales et al. 2005) con vegetación similar; aunque en Ocozocuautla el estudio se realizó en una área conservada (Cuadro 6). Sin embargo, el municipio de Villaflores se caracteriza como una zona productora de ganado y granos básicos, para lo cuál se ocupan grandes extensiones de terreno, lo que posiblemente modifica la riqueza de melolóntidos en la zona; ya que la composición de la entomofauna usualmente se ve influída por las modificaciones que las actividades humanas ejercen en los hábitats naturales, propiciando la disminución de unas especies y el éxito de otras a nivel local y regional (Morón \& Terrón 1988, Van Hook 1994).

En cuanto a la riqueza específica por sitios, se encontró mayor riqueza en las localidades de El Jardín (37 especies) y Francisco Villa (38 especies). Francisco Villa es un sitio que se encuentra aproximadamente a $15 \mathrm{~km}$ del área de amortiguamiento de la reserva de la Biosfera "La Sepultura". Lo que hace suponer que la diversidad es más rica por la cercanía de la reserva; mientras que El Jardín se encuentra entre dos cerros muy conservados lo que posiblemente haya influido en la diversidad de especies y su abundancia.

Los sitios más perturbados como Villa Flores (29 especies), que al ser la cabecera municipal presenta más asentamientos humanos, y Cuauhtémoc (27 especies) con 
grandes extensiones de parcelas dedicadas a la agricultura y la ganadería, resultaron con menor cantidad de especies y menor abundancia.

En general, es probable que los datos de la diversidad encontrada en los terrenos agrícolas estén influenciados por la capacidad de vuelo de los adultos, que pueden dispersarse en distancias amplias en busca de alimento, y posteriomente regresan a refugiarse y/o ovipositar en áreas con vegetación silvestre. Esto se ha observado con Phyllophaga rufotestacea en San Cristóbal de Las Casas, Chiapas, donde sus adultos llegan a alimentarse a parcelas cultivadas pero sus inmaduros no se encuentran en ellas (Pacheco-Flores \& Castro-Ramírez 2005).

Los aspectos fenológicos y ecológicos de los adultos de las especies más abundantes en estas localidades, así como algunos datos sobre la actividad de las larvas edafícolas, serán incorporados en otra publicación que se encuentra en preparación.

\section{AGRADECIMIENTOS}

La investigación se realizó gracias a la aportación económica del programa de apoyo de tesis de maestría (PATM) y la línea de Diversidad en Sistemas de Cultivos de ECOSURUnidad San Cristóbal. Al CONACYT por la beca otorgada al primer autor. A los agricultores Raquel Padilla, Elías Sánchez, Joaquín Altamirano, Tavín Gómez, Julio Nuricumbo y Guadalupe Gómez Pérez por su apoyo en el trabajo de campo. A la Ing. Concepción Ramírez Salinas la revisión del material entomológico. Al Quím. Miguel López Anaya, Biol. Manuel de J. Anzueto y al Tec. Manuel de J. Gutiérrez su colaboración durante el trabajo de laboratorio y campo. A Henry E. Castañeda y Miguel Martínez Icó la identificación del material botánico; y a los técnicos Julio César Llanes Monsreal y Adrian Sarabia Rangel la elaboración del mapa.

\section{LITERATURA CITADA}

Alcázar-Ruiz, J.A., A. Morón-Ríos \& M. A. Morón. 2003. Fauna de Coleoptera Melolonthidae de Villa Las Rosas, Chiapas, México. Acta Zool. Mex. (n.s.) 88: 59-86.

Castro-Ramírez, A. E., C. Ramírez-Salinas \& C. Pacheco-Flores. 2003. Escarabajos melolóntidos (Coleoptera) de tres localidades en Los Altos de Chiapas, México. Pp. 394399. In: J. Romero-Nápoles, E. Estrada-Villegas \& A. Equihua-Martínez (Eds.). Entomología Mexicana, vol.2. Sociedad Mexicana de Entomología, México.

Castro-Ramírez, A.E., H. Delfín-González, V. Parra-Tabla. \& M. A. Morón. 2005. Fauna de melolóntidos (Coleoptera: Scarabaeoidea) asociados al maíz (Zea mays L.) en Los Altos de Chiapas, México. Folia Entomol. Mex. 44 (3): 339-365.

Córdova R., M. 2002. Estudio socioeconómico del municipio de Villaflores, Chiapas (monografía de un pueblo en desarrollo). Universidad Autónoma Chapingo, H. Ayuntamiento de Villaflores, Chiapas. 58 p. 
Deloya L., C. 2006. Escarabajos fitófagos del bosque mesófilo de montaña y comunidades derivadas en el centro de Veracruz, México (Coleoptera: Scarabaeoidea). Pp. 81-98. In: A. E. Castro-Ramírez, M.A. Morón \& A. Aragón (Eds.). Diversidad, importancia y manejo de escarabajos edafícolas. Publicación especial de El Colegio de la Frontera Sur, la Fundación PRODUCE Chiapas A. C. y la Benemérita Universidad Autónoma de Puebla, México.

Deloya, C. \& M. A. Morón. 1998. Nuevas especies de Phyllophaga Harris (Coleoptera: Melolonthidae) de Morelos, Puebla y Chiapas, México. Folia Entomol. Méx. 104: 109119.

Gómez y Gómez, B. 1998. Listado preliminar de los escarabajos (Coleoptera: Melolonthidae) de la reserva "El Ocote", Chiapas, México. Pp: 427-431. In: Memorias del XXXIII Congreso Nacional de Entomología, Acapulco, Guerrero. Sociedad Mexicana de Entomología, México.

Gómez y Gómez, B. \& E. R. Chamé. 2003. Primeros registros de Goniophileurus femoratus y Sisyphus mexicanus para Chiapas, México. (Coleoptera: Scarabaeioidea). Folia Entomol. Mex. 42 (1): 103-104.

Gómez y Gómez, B. \& V. Galdamez E. 2001. Nuevos registros de Melolonthidae (Coleoptera: Scarabaeoidea) para Chiapas, México. Folia Entomol. Mex. 40 (2):279-280.

Gómez y Gómez, B. y J. Morales-Morales. 2003. Trigonopeltastes geometrica Schaum, 1841 en Chiapas, México (Coleoptera: Melolonthidae: Trichinae). Folia Entomol. Mex. $42(2): 291-292$.

LAIGE-ECOSUR, 2006. Base de datos digitales. El Colegio de la Frontera Sur (ECOSUR). San Cristóbal de Las Casas, Chiapas, México.

Méndez-Aguilar, M. J., A. E. Castro-Ramírez, R. Alvarado-Barrantes, C. PachecoFlores \& C. Ramírez-Salinas. 2005. Eficacia de dos tipos de recolectas para registrar la diversidad de melolóntidos nocturnos (Coleoptera: Scarabaeoidea). Acta Zool. Mex. (n.s.) 21(3):109-124.

Micó, E., B. Gómez \& E. Galante. 2006. The Mesoamerican genus Yaaxkumukia: Biogeography and descriptions of new species (Coleoptera: Rutelidae). Ann. Entomol. Soc.Am. 99 (1): 1-6.

Morales-Morales, C. J., M. A. Rosales-Esquinca, P. Ponce-Díaz, P., R. C. GutiérrezHernández, R. R. Quiroga-Madrigal \& J. L. Zuart-Macías. 2005. Melolóntidos (Coleoptera: Scarabaeoidea) de la zona de conservación ecológica Laguna Bélgica, Ocozocuautla, Chiapas, México. Pp.144-148. In: Entomología Mexicana vol. 4. Sociedad Mexicana de Entomología, México.

Morón, M.A. 1990. Rutelini 1. Los coleópteros del mundo, vol. 10. Sciences Nat. Venette, France.

Morón, M.A. 1995a. Fenología y hábitos de los Cetoniinae (Coleoptera: Melolonthidae) en la región de Xalapa-Coatepec, Veracruz, México. G. it. Ent. 7: 317-332.

Morón, M. A. 1995b. Review of the Mexican species of Golofa Hope (Coleoptera: Melolonthidae: Dynastinae). Coleopts. Bull. 49(4): 343-386

Morón, M. A. 1995c. Larva and pupa of Archedinus relictus Morón \& Krikken (Coleoptera: Melolonthidae, Trichinae, Incaini). Pan-Pac. Ent. 71(4): 237-244. 
Morón, M.A. 1997. Inventarios faunísticos de los Coleoptera Melolonthidae Neotropicales con potencial como bioindicadores. G. it. Ent. 8: 265-274.

Morón, M. A. 1999. Four new species of Phyllophaga Harris (Coleoptera: Melolonthidae, Melolonthinae) from Southeastern México. Coleopts. Bull. 53(4): 377-387.

Morón, M. A. 2001. New and rare species of Phyllophaga (s.str.) from México (Coleoptera: Melolonthidae, Melolonthinae). Pan-Pac. Ent. 77(3): 168-189.

Morón, M. A. 2003a. Revisión of the Phyllophaga s.s. schizorhina species group (Coleoptera: Melolonthidae: Melolonthinae). Can. Entomol. 135: 213-302.

Morón, M. A. 2003b. Diversidad, distribución e importancia de las especies de Phyllophaga Harris en México (Coleoptera: Melolonthidae). Pp: 1-27. In: A. Aragón, M. A. Morón \& A. Marín (Eds.). Estudios sobre coleópteros del suelo en América. Publicación especial de la Benemérita Universidad Autónoma de Puebla, México.

Morón, M. A. \& E. B. Cano. 2000. Three new species of Phyllophaga (s.str.) group rostripyga (Coleoptera: Melolonthidae, Melolonthinae) from Mexico and Guatemala. Pan-Pac. Ent. 76(4): 234-242.

Morón, M. A. \& C. Deloya, 1991. Los coleópteros lamelicornios de la reserva de la Biosfera "La Michilía", Durango, México. Folia Entomol. Mex. 81: 209-283

Morón, M. A., C. Deloya, A.Ramírez-Campos \& S. Hernández-Rodríguez. 1998. Fauna de Coleoptera Lamellicornia de la región de Tepic, Nayarit, México. Acta Zool. Mex. (n.s.) 75: 73-116.

Morón, M. A. \& Paucar-Cabrera, A. 2003. Larvae and pupae of species of the genus Macraspis (Coleoptera: Scarabaeidae: Rutelinae: Rutelini). The Canadian Entomologist 135: 467-491.

Morón, M.A., B. C. Ratcliffe \& C. Deloya. 1997. Atlas de los escarabajos de México, Coleoptera: Lamellicornia. Vol. I Familia Melolonthidae. Sociedad Mexicana de Entomología A. C. y CONABIO. México.

Morón, M.A. \& E. G. Riley. 2005. Four new species of Phyllophaga from México and Central America (Coleoptera: Scarabaeidae: Melolonthinae). Coleops. Bull. 59 (3): 391-399.

Morón, M.A. \& Terrón, R. 1988. Entomología práctica. Publicación no. 22. Instituto de Ecología, A. C. México D. F.

Morón, M.A., F. J. Villalobos \& C. Deloya. 1985. Fauna de coleópteros Lamellicornios de Boca de Chajul, Chiapas, México, Folia Entomol. Mex. 66: 57-118.

Morón-Ríos, A. \& M. A. Morón. 2001. La fauna de Coleoptera Melolonthidae de la reserva de la biosfera "El Triunfo", Chiapas, México. Acta Zool. Mex. (n.s.) 84: 1-25.

Orozco Z., M. A. 2002. Geohistoria de Chiapas. Ediciones y sistemas especiales. Chiapas, México.

Palacios-Ríos, M., V. Rico-Gray \& E. Fuentes. 1990. Inventario preliminar de los Coleoptera Lamellicornia de la zona de Yaxchilán, Chiapas, México. Folia Entomol. Mex. 78: 49-60.

Pacheco-Flores, C. \& A. E. Castro-Ramírez. 2005. Primer registro de Phyllophaga (Phytalus) rufotestacea (Moser, 1918) (Coleoptera: Scarabaeoidea) para México y Chiapas. Acta Zool. Mex. (n.s.) 21(2): 157-158.

Rzedowski, J. 1978. Vegetación de México. Limusa. México. 
SEMARNAP. 1999. Programa de manejo Reserva de la Biosfera La Sepultura, México. Instituto Nacional de Ecología, SEMARNAP, México.

Thomas, B. D. 1993. Scarabaeidae (Coleoptera) of the Chiapanecan forest: A faunal survey and chorographic analysis. Coleopts. Bull. 47(4): 363-408.

Van Hook, T. 1994. The conservation challenge in agriculture and the role of entomologist. Florida Entomol. 77 (1): 42-73.

\begin{abstract}
ANEXO 1
Especies de Melolonthidae citadas para el estado de Chiapas (366), de acuerdo a la revisión de literatura cotejada por Cutberto Pacheco y M.A. Morón; más dos registros obtenidos en este estudio para el estado (368). Los números entre paréntesis corresponden con las referencias citadas al final del anexo.
\end{abstract}

ESPECIES DISTRIBUCIÓN

Subfamilia: Cetoniinae

Amithao cavifrons (Burmeister, 1847) (5) (23) (24) (26)

Ocosingo, Reserva El Ocote, Yaxchilán, Boca de Chajul, Bonampak, Laguna Bélgica

Amithao erythropus (Burmeister, 1842) (24)

Amithao pyrrhonotum (Burmeister, 1842) (27)

Amithao haematopus (Schaum, 1848) (5) (11) (24)

Argyripa porioni Arnaud, 1988 (24)

Ocosingo, Ocozocuautla

Ocozocuautla

Argyripa moroni Arnaud, 1988 (24)

Ocosingo, Reserva El Ocote, Laguna Bélgica

Ocosingo

Balsameda pulverulenta (Burmeister, 1842) (24)

Ocosingo, Cacaohatán

Tuxtla Gutiérrez, Cañón del Sumidero, El Aguacero

Cotinis mutabilis (Gory \& Percheron, 1833) (5) (11)

Reserva El Ocote, Laguna Bélgica, Tuxtla Gutiérrez, El Sumidero, Comitán, Palenque, Simojovel, Ocozocuautla, Rizo de Oro

Cotinis fuscopicea Goodrich, $1966^{(11)}$ Laguna Bélgica, El Sumidero, Ocozocuautla

Cotinis subviolacea (Gory \& Percheron, 1833) (24)

Cotinis pauperula (Gory \& Percheron, 1847) (11)

Euphoria basalis (Gory \& Percheron, 1833) (24)

Euphoria biguttata (Gory \& Percheron, 1833) (24)

Euphoria candezei Janson, 1875 (5) (11) (23) (24)

Lagos de Montebello, Santa Rosa, Bonampak Laguna Bélgica, El Aguacero, Rizo de Oro, El Sumidero, Tuxtla Gutiérrez, Ocozocuautla San Cristóbal de Las Casas

El Sumidero, Rizo de Oro, Tuxtla Gutiérrez, La Trinitaria

Ocosingo, Comitán, Reserva El Ocote, Laguna Bélgica, Boca de Chajul, Palenque, Lagos de Montebello, Ocozocuautla

Euphoria fulveola Bates, 1889 (27)

Euphoria geminata (Chevrolat, 1835) (24)

No confirmada

El Sumidero 
Pacheco Flores et al.: Fauna de escarabajos melolóntidos (Coleoptera: Scarabeoidea) en Chiapas, México

Continúa

\begin{tabular}{|c|c|}
\hline ESPECIES & DISTRIBUCIÓN \\
\hline Euphoria iridescens Schaum, 1841 (27) & Tuxtla Gutiérrez, El Sumidero \\
\hline Euphoria leucographa Gory \& Percheron, 1833 (24) (11) & Laguna Bélgica \\
\hline Euphoria lineoligera Blanchard, 1850 (27) & El Sumidero, El Aguacero \\
\hline Euphoria leseuri Gory \& Percheron, 1833 (27) & $\begin{array}{l}\text { El Aguacero, El Sumidero, Laguna Bélgica, } \\
\text { Tuxtla Gutiérrez, Chincultic }\end{array}$ \\
\hline Euphoria dimidiata (Gory \& Percheron, 1833) (11) & $\begin{array}{l}\text { Laguna Bélgica, El Sumidero, Tuxtla Gutiérrez, } \\
\text { La Trinitaria, Rizo de Oro, Ocozocuautla }\end{array}$ \\
\hline Euphoria pulchella (Gory \& Percheron, 1833) (11) & Laguna Bélgica, Simojovel, El Sumidero \\
\hline Euphoria sepulchralis (Fabricius) 1801 (27) & $\begin{array}{l}\text { Tuxtla Gutiérrez, El Sumidero, Chicoasén, San } \\
\text { Cristóbal de Las Casas, El Chorreadero }\end{array}$ \\
\hline Euphoria subtomentosa Mannerheim, 1837 (27) & $\begin{array}{l}\text { El Sumidero, Simojovel, Rizo de Oro, Tuxtla } \\
\text { Gutiérrez }\end{array}$ \\
\hline Euphoria westermani (Gory \& Percheron, 1833) (26) & Yaxchilán \\
\hline Genuchinus quinquenotata (Westwood) (27) & El Sumidero \\
\hline Guatemalica marginicollis (Burmeister, 1842) (27) & Tuxtla Gutiérrez? \\
\hline Gymnetis difficilis Burmeister 1842 (27) & Laguna Bélgica, Agua Azul \\
\hline Gymnetis sallei Schaum, 1849 (27) & Ocozocuautla, Sumidero, Cinco Cerros \\
\hline Gymnetis bajula radicollis Burmeister (24) (26) & Huixtla, Yaxchilán \\
\hline Gymnetis stellata (Latreille, 1833) (24) & $\begin{array}{l}\text { Tapachula, Simojovel, Lagos Montebello, } \\
\text { Tuxtla Gutiérrez, Ocozocuautla }\end{array}$ \\
\hline $\begin{array}{l}\text { Gymnetis pardalis (Gory \& Percherón, 1833) (24) } \\
\text { Hologymnetis kinichahau Ratcliffe \& }\end{array}$ & Comitán \\
\hline Deloya, 1992 (20) (27) & $\begin{array}{l}\text { San Cristóbal, Rincón Chamula, Motozintla, } \\
\text { Santa Rosa, San Quintín }\end{array}$ \\
\hline Hologymnetis cinerea (Gory \& Percheron, 1833) (5) (11) & $\begin{array}{l}\text { Reserva El Ocote, Laguna Bélgica, Lagos } \\
\text { Montebello, El Sumidero, Tuxtla Gutiérrez, } \\
\text { Ocozocuautla }\end{array}$ \\
\hline Hologymnetis margaritis Raccliffe \& Deloya, 1992 (24) & $\begin{array}{l}\text { Ciudad Cuauhtémoc, Tapachula, El Aguacero, } \\
\text { Rizo de Oro }\end{array}$ \\
\hline Hoplopyga liturata (Olivier, 1789) (24) & Comitán, Laguna Bélgica, Simojovel \\
\hline Hoplopyga ocellata (Gory \& Percheron, 1833 ) (11) & Laguna Bélgica \\
\hline Marmarina maculosa (Olivier, 1789) (6) & El Suspiro, municipio de Berriozábal \\
\hline \multicolumn{2}{|l|}{ Paragymnetis flavomarginata poecila } \\
\hline Schaum, 1848 (24) (26) & Huixtla, Yaxchilán \\
\hline \multicolumn{2}{|l|}{ Paragymnetis hebraica difficilis } \\
\hline Burmeister, 1842 (20) (24) (26) & Comitán, Ocosingo, Yaxchilán, El Triunfo \\
\hline
\end{tabular}

Subfamilia Dynastinae

\begin{tabular}{ll}
\hline Ancognatha falsa Arrow, 1911 (24) & Simojovel, Solistahuacan \\
Archophileurus simplex (Bates, 1888) (1) & Villa Las Rosas
\end{tabular} 
Continúa

\begin{tabular}{|c|c|}
\hline ESPECIES & DISTRIBUCIÓN \\
\hline Aspidolea singularis Bates, 1888 (5) (11) (23) (24) (26) & $\begin{array}{l}\text { Yaxchilán, Tapilula, Tapachula, Huixtla, } \\
\text { Ocosingo, Reserva El cote, Laguna Bélgica, } \\
\text { Boca de Chajul, Palenque }\end{array}$ \\
\hline Aspidolea fuliginea (Burmeister, 1847) (24) & Ocosingo, Palenque, Yaxchilan \\
\hline Bothynus quadridens Taschenberg, 1870 (27) & Ocosingo, Simojovel \\
\hline Coelosis biloba (Linné, 1767) (1) (11) (23) (24) (26) & $\begin{array}{l}\text { Trinitaria, Zanatepec, Boca de Chajul, Laguna } \\
\text { Bélgica, Yaxchilán, El Chorreadero, Villaflores, } \\
\text { Simojovel, Palenque, Cinco Cerros }\end{array}$ \\
\hline Cyclocephala aequatoria Endrodi, 1963 (24) (26) & Yaxchilán \\
\hline
\end{tabular}

Cyclocephala alexi Ratcliffe and Delgado, 1988 (2) (3) (9) (24)

Lago Montebello, Jitotol, Chilil, Selva Negra, Navil, Tzunum, Balun Canal, Tenejapa, Majosik, Piedra Escrita, San Cristóbal de Las Casas, Tzizcao, Comitán

Cyclocephala amazona (Linné, 1767) (1) (11) (24) (26)

Berriozabal, Chiapa de Corzo, Palenque, Tuxtla Gutiérrez, Ocozocuautla, Coapilla, Boca de Chajul, Laguna Bélgica, Yaxchilán, Villa Las Rosas

Cyclocephala amblyopsis Bates, 1888 (24) (20)

Cyclocephala carbonaria Arrow, 1911 (24)

No confirmada

No confirmada

Cyclocephala complanata Burmeister, 1847 (1) (5) (24) (26)

Albino Corzo, Ocozocuautla, Palenque, Trinitaria, Huixtla, Acapetahua, Reserva El Ocote, Yaxchilán, Villa Las Rosas

Cyclocephala concolor Burmeister, 1847 (24) Cyclocephala curta Bates, 1888 (24) No confirmada

No confirmada

Cyclocephala discolor Herbst, 1792 (20)

El Triunfo

Cyclocephala deceptor Casey, 1915 (27)

El Aguacero, El Chorreadero, Cintalapa, Chicoasén, Ocozocuautla

Cyclocephala erotylina Arroz, 1914 (24) Cyclocephala fasciolata Bates, 1888 (24) Cyclocephala fulgurata Burmeister, 1847 (24) Cyclocephala freudei Endrodi, 1963 (23) (27) Huixtla Ocosingo, Pichucalco, Tapilula Lagos de Montebello, Solosuchiapa Chajul Cyclocephala gravis Bates, 1888 (5) (24)

Reserva El Ocote, Laguna Bélgica, Bonampak, Simojovel, Palenque, Cinco Cerros

Cyclocephala guttata Bates, 1888 (23) (24) Cyclocephala jalapensis Casey, 1915 (27) Cyclocephala landini Endrodi, 1964 (23) (26) Boca de Chajul, El Aguacero, El Chorreadero Chilil

Yaxchilán, Boca de Chajul

Cyclocephala lunulata Burmeister, 1847 (1) (3) (5) (23) (24) (26)

Reserva El Ocote, Yaxchilán, Boca de Chajul, El Aguacero, Catazaja, Palenque, El Chorreadero, Laguna Bélgica, Berriozábal, Ocozocuautla, Chicoasén, Yalumá 
Pacheco Flores et al.: Fauna de escarabajos melolóntidos (Coleoptera: Scarabeoidea) en Chiapas, México

Continúa

\begin{tabular}{|c|c|}
\hline ESPECIES & DISTRIBUCIÓN \\
\hline Cyclocephala mafaffa Burmeister, 1847 (1) (11) (20) (24) & $\begin{array}{l}\text { Laguna Bélgica, El Chorreadero, Cinco Cerros, } \\
\text { Rizo de Oro, Ocozocuautla, Selva Negra, Villa } \\
\text { Las Rosas }\end{array}$ \\
\hline Cyclocephala marginicollis Arrow, 1902 (24) (26) & Yaxchilán \\
\hline Cyclocephala melanocephala (Fabricius, 1775) (11) (23) (24) & $\begin{array}{l}\text { Laguna Bélgica, Boca de Chajul, Palenque, El } \\
\text { Aguacero, Ocozocuautla, Cinco Cerros, } \\
\text { Cuxtepeques, Pichucalco }\end{array}$ \\
\hline Cyclocephala ovulum Bates, 1888 (27) & $\begin{array}{l}\text { El Aguacero, Ocozocuautla, Villaflores, Cinco } \\
\text { Cerros }\end{array}$ \\
\hline Cyclocephala picta Burmeister, 1847 (3) (24) & Selva Negra, Piedra Escrita \\
\hline Cyclocephala pubescens Burmeister, 1847 (24) & Palenque \\
\hline Cyclocephala prolongata Arrow, 1902 (23) (26) & Boca de Chajul, Yaxchilán \\
\hline Cyclocephala sanguinicollis Burmeister, 1847 (5) (23) (24) (26) & Reserva El Ocote; Yaxchilán, Boca de Chajul \\
\hline Cyclocephala sexpunctata Castelnau, 1840 (5) (24) & $\begin{array}{l}\text { Tapachula, Huixtla, Cacaohatán, Jitotol, } \\
\text { Comitán, Simojovel, Palenque, Ocosingo, } \\
\text { Reserva El Ocote }\end{array}$ \\
\hline Cyclocephala signaticollis Burmeister, 1847 (27) & El Aguacero, El Chorreadero, Chicoasén \\
\hline Cyclocephala sororia Bates, 1888 (24) & No confirmada \\
\hline Cyclocephala sparsa Arrow, 1902 (5) (11) (24) & $\begin{array}{l}\text { Reserva El Ocote, Laguna Bélgica, Villa Flores, } \\
\text { Chicoasén, Ocozocuautla, El Aguacero }\end{array}$ \\
\hline Cyclocephala stictica Burmeister, 1847 (1) (5) (11) (24) (26) & $\begin{array}{l}\text { Reserva El Ocote, Laguna Bélgica, Yaxchilán, } \\
\text { El Aguacero, Laguna Bélgica, Villaflores, } \\
\text { Simojovel, Ocozocuautla, Cuxtepeques, } \\
\text { Palenque, Solosuchiapa }\end{array}$ \\
\hline Cyclocephala testacea ovulum Bates, 1888 (24) & Cintalapa, Ocozocuautla \\
\hline Cyclocephala tutilina Burmeister, 1847(24) & No confirmada \\
\hline Cyclocephala weidneri Endrodi, 1964 (24) (20) & El Triunfo \\
\hline Dyscinetus dubius (Olivier, 1789) (24) & $\begin{array}{l}\text { Tuxtla Gutiérrez, Ocosingo, } \\
\text { Tapachula, Laguna Bélgica }\end{array}$ \\
\hline Dyscinetus laevipunctatus Bates, 1888 (23) & $\begin{array}{l}\text { Tuxtla Gutiérrez, Ocosingo, Boca de Chajul, } \\
\text { Simojovel }\end{array}$ \\
\hline Dynastes hyllus Chevrolat, 1843 (24) & Simojovel, Selva Negra, Albino Corzo \\
\hline Dynastes maya Hardy, 2000 & $\begin{array}{l}\text { Ocuilapa, Ocozocuautla, Laguna Bélgica, Cinco } \\
\text { Cerros }\end{array}$ \\
\hline Enema endymion Chevrolat, 1843 (1) (2) (5) (11) (24) & $\begin{array}{l}\text { Ocozocuautla, Tuxtla Gutiérrez, Chiapa de } \\
\text { Corzo, Berriozábal, Reserva El Ocote, Laguna } \\
\text { Bélgica, Simojovel, El Aguacero, Tzunum }\end{array}$ \\
\hline Enema pan (Fabricius, 1775) (24) & Ocosingo \\
\hline Euetheola bidentata Burmeister, 1847 (1) (5) (11) (27) & $\begin{array}{l}\text { Laguna Bélgica, El Aguacero, Ocozocuautla, } \\
\text { Cinco Cerros, El Chorreadero, Villa Las Rosas }\end{array}$ \\
\hline Euetheola humilis Burmeister, 1847 (24) & Tuxtla Gutiérrez, Ocozocuautla \\
\hline
\end{tabular}


Continúa

\begin{tabular}{|c|c|}
\hline ESPECIES & DISTRIBUCIÓN \\
\hline Goniophileurus femoratus (Burmeister, 1847) (7) & Barrio Nueva Morelia, eijido San Juan Panamá \\
\hline Golofa imperialis Thomson, 1858 (13) (24) & $\begin{array}{l}\text { Teopisca, Chiapas de Corzo, Zinacantan, } \\
\text { Ocozocuautla, Comitán, Albino Corzo, Cañón } \\
\text { El Sumidero, Finca Liquidambar }\end{array}$ \\
\hline Golofa imbellis Bates, 1888 (27) & San Cristóbal \\
\hline Golofa pizarro Hope, 1837 (13) (20) (24) & $\begin{array}{l}\text { Berriozábal, El Suspiro, Ocosingo, Rayón, } \\
\text { Laguna Bélgica, El Sumidero, Simojovel, El } \\
\text { Triunfo }\end{array}$ \\
\hline Golofa tersander Burmeister, 1847 (5) (11) (13) (23) (24) & $\begin{array}{l}\text { Boca de Chajul, El Bosque, Frontera Corozal, } \\
\text { Ocozocuautla, Bonampak, Yajalón, Reserva El } \\
\text { Ocote, Laguna Bélgica, Ocosingo }\end{array}$ \\
\hline Heterogomphus chevrolati Burmeister, 1847 (23) (24) & $\begin{array}{l}\text { Ocosingo, Simojovel, La Trinitaria, Boca de } \\
\text { Chajul, Bochil, Lagos de Montebello, Selva } \\
\text { Negra }\end{array}$ \\
\hline Heterogomphus pehlkei (Kolbe, 1906) (20) (24) & $\begin{array}{l}\text { San Antonio, Tapachula, Cinco Cerros, El } \\
\text { Aguacero, Laguna Bélgica }\end{array}$ \\
\hline Hemiphileurus dejeani (Bates, 1888) (1) (5) (11) (23) (24) & $\begin{array}{l}\text { Bochil, Huixtla, Ocosingo, Berriozábal, } \\
\text { Cacaohatán, Reserva El Ocote; Laguna Bélgica, } \\
\text { Boca de Chajul, Villa Las Rosas }\end{array}$ \\
\hline Hemiphileurus laevicauda (Bates, 1888) (24) & Tapachula \\
\hline Hemiphileurus microps Burmeister, 1847 (1) (23) & $\begin{array}{l}\text { Boca de Chajul, Simojovel, Bochil, Ocosingo y } \\
\text { Berriozábal }\end{array}$ \\
\hline Hemiphileurus punctatostriatus Prell, 1914 (5) (23) & Reserva El Ocote, Boca de Chajul \\
\hline \multicolumn{2}{|c|}{ Homophileurus quadrituberculatus (Beauvois, 1806) (24) Laguna Bélgica } \\
\hline Homophileurus tricuspis Prell, 1914 (5) (23) (24) (26) & $\begin{array}{l}\text { Yaxchilán, Boca de Chajul, Reserva El Ocote, } \\
\text { Laguna Bélgica, Simojovel, Ocozocuautla }\end{array}$ \\
\hline Ligyrus cicatricosus Prell, 1937 (27) & El Aguacero, Villaflores \\
\hline Ligyrus nasutus (Burmeister, 1847) (1) (11) (24) & $\begin{array}{l}\text { Tuxtla Gutiérrez, Comitán, Ocozocuautla, } \\
\text { Motozintla, Chiapa de Corzo, Laguna Bélgica, } \\
\text { El Aguacero, Villaflores, Villa Las Rosas }\end{array}$ \\
\hline Ligyrus b. bituberculatus (Beauvois, 1805) (23) (24) & $\begin{array}{l}\text { Palenque, Ocosingo, Boca de Chajul, } \\
\text { Villaflores, Cinco Cerros, Ocozocuautla, Puerto } \\
\text { Arista }\end{array}$ \\
\hline Ligyrus laevicollis Bates, 1888 (24) & Tuxtla Gutiérrez, Cinco Cerros, Puerto Arista \\
\hline Ligyrus gyas Erichson, 1848 (23) (24) & Ocosingo, Boca de Chajul \\
\hline Ligyrus sallei Bates, 1888 (1) (3) (5) (11) & $\begin{array}{l}\text { Simojovel, Albino Corzo, Coapilla, } \\
\text { Ocozocuautla, Tuxtla Gutiérrez, Palenque, } \\
\text { Chicoasén, Reserva El Ocote, Laguna Bélgica, } \\
\text { El Aguacero, Teopisca, El Madronal, San } \\
\text { Cristóbal de Las Casas, Yalumá, Villa Las Rosas }\end{array}$ \\
\hline
\end{tabular}


Pacheco Flores et al.: Fauna de escarabajos melolóntidos (Coleoptera: Scarabeoidea) en Chiapas, México

Continúa

\begin{tabular}{l}
\hline ESPECIES \\
Megasoma elephas Fabricius, 1775 (5) (23) (24) \\
Mimeoma acuta Arrow, 1902 (23) (24) \\
Orizabus clunalis (LeConte, 1856) (2) (9) (24) \\
Orizabus batesi Prell, 1914 (24) \\
Orizabus rubricollis Prell, 1914 (24) \\
Orizabus tuberculatus Prell, 1914 (24) \\
Phileurus didymus (Linné, 1758) (1) (23) (24) (26)
\end{tabular}

Phileurus truncatus (Beauvois, 1807) (24)

Phileurus valgus (Olivier, 1789) (5) (11) (23) (24)

Phileurus angustatus Kolbe, 1910 (24) (27)

Phileurus excavatus Prell, 1911 (24)

Podischnus agenor (Olivier, 1789) (23) (24)

Spodistes mniszechi (Thomson, 1860) (5) (11) (23) (24) (26)

Spodistes monzoni Warner, 1992 (1) (24)

Stenocrates difficilis Endrodi, 1966 (23) (24)

Stenocrates duplicatus Endrodi, 1967 (23)

Stenocrates laevicollis Kirsch, 1870 (24)

Strategus aloeus Linné, 1758 (1) (3) (5) (9) (11) (20)

Strategus longichomperus Ratcliffe, 1976 (5) (23) (26)

Strategus hipposiderus Ratcliffe, 1976 (23) (24) (26)

Strategus jugurtha Burmeister, 1847 (24)

Xyloryctes lobicollis Bates, 1888 (1) (2) (3) (20) (24)

Xyloryctes thestalus Bates, 1888 (24)

\section{DISTRIBUCIÓN}

Tuxtla Gutiérrez, Tapachula, Boca de Chajul, Reserva El Ocote, Yaxchilán, El Aguacero, Cacahoatán, Palenque, Arriaga, Simojovel

Ocosingo, Boca de Chajul

Tapilula, Tzunum, San Cristóbal de Las Casas y Selva Negra

San Cristóbal de Las Casas

Tecpatan

San Cristóbal de Las Casas

Tuxtla Chico, Tapachula, Palenque, Ocozocuautla, Yaxchilán, Boca de Chajul, Laguna Bélgica, Cacahoatán, Simojovel

Ocozocuautla

Tuxtla Gutiérrez, Reserva El Ocote, Ocozocuautla, Laguna Bélgica, Boca de Chajul, El Aguacero, Cinco Cerros

Laguna Bélgica

Tapachula, Simojovel, Laguna Bélgica

Boca de Chajul

Boca de Chajul, Parque Laguna Bélgica, Reserva El Ocote, Laguna Bélgica, Yaxchilán, Ocozocuautla, Ixtapa

Huixtla, Villa Las Rosas

Palenque, Boca de Chajul, Simojovel

Boca de Chajul

Boca de Chajul, Laguna Bélgica

San Cristóbal de Las Casas, Reserva El Ocote, Laguna Bélgica, El Aguacero, Villaflores, Tuxtla Gutiérrez, Ocozocuautla, Chiapa de Corzo, Comitán, Simojovel, Aguacatenango, Tenejapa, Piedra Escrita y Teopisca

Palenque, Boca de Chajul, Reserva El Ocote, Yaxchilán, Palenque

Ocosingo, Yaxchilán, Boca de Chajul

Ocozocuautla, Cacaohatán, Tuxtla Gutiérrez, El Aguacero, Villaflores, Rizo de Oro

Tuxtla Gutiérrez, Simojovel, Tapachula, Trinitaria, Ocosingo, Huixtla, El Bosque, Teopisca, Tapilula, Coapilla, Lagos Montebello, Tzunum, El Triunfo, Aguacatenango San Cristóbal de Las Casas 
Continúa

\begin{tabular}{l} 
ESPECIES \\
\hline Xyloryctes ensifer Bates, 1888 (20) (24) \\
Xyloryctes teuthras Bates, 1888 (2) (3) (9) (20) (24) \\
Familia Melolonthinae \\
Ceraspis mexicana Harold, 1863 (11) (23) (24) \\
Ceraspis pilatei Harold, 1863 (23) (26) (24) \\
Diplotaxis alutacea Bates, 1889 (27) \\
Diplotaxis angustula Moser, 1918 (5) (24) (27)
\end{tabular}

Diplotaxis carinifrons Bates, 1889 (5) (11) (23) (24) (27)

Diplotaxis cavifrons (Moser, 1918) (3) (24) (27) (29)

Diplotaxis commixta Vaurie, 1960 (1) (24) (27)

Diplotaxis consentanea Bates, 1887 (1)

Diplotaxis crinigera Bates, 1888 (24) (27)

Diplotaxis crucis Vaurie, 1960 (24) (27)

Diplotaxis denigrata Bates, 1889 (3) (24) (27)

Diplotaxis hallei Vaurie, 1958 (presente estudio)

Diplotaxis hirsuta Vaurie, 1958 (1) (5) (24) (27)

Diplotaxis jacala Vaurie, 1958 (1)

Diplotaxis macrotarsus Vaurie, 1960 (24) (27)

Diplotaxis maya Vaurie, 1958 (11) (24)

Diplotaxis megapleura Vaurie, 1960 (24) (27)

Diplotaxis mistura Vaurie, 1960 (24) (27)

Diplotaxis microchele Vaurie, 1960 (27)

\section{DISTRIBUCIÓN}

Albino Corzo, San Cristóbal de Las Casas, El Triunfo

San Cristóbal de Las Casas, Comitán, La Independencia, Huixtla, Suchiapa, Tzunum, Juznajab, Piedra Escrita, Tenejapa, Teopisca, Yalumá, El Triunfo

Boca de Chajul, Laguna Bélgica, Simojovel, Palenque

Boca de Chajul, Yaxchilán y Solosuchiapa

Lagos de Montebello y Teopisca

Reserva El Ocote, Tuxtla Gutiérrez, Tonalá, El Chorreadero, Chicoasén, Huixtla, Ocozocuautla, El Aguacero

Reserva El Ocote, Laguna Bélgica, Boca de Chajul, La Trinidad, Palenque, El Aguacero, Simojovel

San Cristóbal de Las Casas, Teopisca, Chilil.

Cinco Cerros, Arriaga, Tuxtla Gutiérrez, Villa

Las Rosas, Ocozocuautla, El Aguacero y Berriozábal

Villa Las Rosas

Jitotol, El Chorreadero, Chicoasén, Cuxtepeques, Ocozocuautla, El Aguacero, Las Rosas

Las Cruces, Berriozabal

Cinco Cerros, El Aguacero, Berriozábal, Chicoasén, Tuxtla Gutiérrez, Cintalapa, Yalumá Francisco Villa, Cuauhtémoc, Villaflores y El Jardín.

Berriozabal, Reserva El Ocote, Yaxchilán, Laguna Bélgica, Palenque, El Aguacero, Ocozocuautla, Yaxchilán, Villa Las Rosas

Villa Las Rosas

Chicoasén, Berriozábal

Laguna Bélgica, Tuxtla Gutiérrez, El Chorreadero, Ocozocuautla, Chicoasén, Cinco Cerros, Berriozábal y El Aguacero

Cinco Cerros, Río San Gregorio

Las Cruces

Cinco Cerros 
Pacheco Flores et al.: Fauna de escarabajos melolóntidos (Coleoptera: Scarabeoidea) en Chiapas, México

Continúa

\begin{tabular}{ll}
\hline \multicolumn{1}{c}{ ESPECIES } & \multicolumn{1}{c}{ DISTRIBUCION } \\
\hline Diplotaxis microtichia Moser, 1912 (27) & Cuxtepeques, Chicoasén \\
Diplotaxis ohausi Moser, 1921 (24)(27) & Simojovel \\
Diplotaxis patyvaurieae Delg., $1990(24)(27)$ & Huixtla \\
Diplotaxis poropyge Bates, 1887 (24) (27) & Teopisca, Ocozocuautla, El Aguacero, \\
Diplotaxis puberea Bates, 1887 (2) (24) (2) & Tuxtla Gutiérrez, Teopisca, San Cristóbal de Las \\
& Casas, Cuxtepeques, Navil, Pacvilná, Tzunum, \\
& Piedra Escrita, Aguacatenango
\end{tabular}

Diplotaxis rugosipennis Blanch., 1851 (20)

El Triunfo

Diplotaxis puncticollis (Moser, 1918) (2)(3)(9)(11)(24) Laguna Bélgica, San Cristóbal de Las Casas, Tuxtla Gutiérrez, El Chorreadero, Teopisca, Jitotol, Simojovel, Navil, Piedra Escrita, Yalumá, Aguacatenango

Diplotaxis rita Vaurie, 1958 (24) (27) El Aguacero, Santa Rita

Diplotaxis simplex Blanch., 1851 (1) (24) (27)

Diplotaxis simillima Moser, 1921 (1)

Diplotaxis tehuana Vaurie, 1960 (27)

Diplotaxis trapezifera Bates, 1887 (24) (27)

Diplotaxis yucateca Vaurie, 1960 (24) (27)

Hoplia argyritis Bates, 1887 (27)

Hoplia inops Bates, 1887 (27)

Hoplia festiva Burmeister, 1844 (27)

Hoplia subcostata Bates, 1887 (20)

Hoplia squamifera Burmeister, 1844 (1)

Isonychus ocellatus Burmeister, 1855 (11)

Isonychus neglectus (Moser, 1918) (2) (3)

Isonychus pictus Sharp, 1877 (27)

Isonychus vittatus Burmeister, 1855 (20)

Macrodactylus costulatus Bates, 1887 (27)

Macrodactylus dimidiatus Guérin, 1884 (24) (27)

Macrodactylus championi Bates, 1887 (20) (24)

Macrodactylus fulvescens Bates, 1887 (3) (11) (24)

Macrodactylus lineatus Chevrolat, 1834 (27)

Macrodactylus nigripes Bates, 1887 (3) (24)

Macrodactylus rufescens (Bates, 1887) (27)

Polyphylla petiti (Guerin, 1830) (1) (3) (20) (27)

Laguna Bélgica, Ocosingo, El Chorreadero, El Aguacero, Villa Las Rosas

Villa Las Rosas

Cinco Cerros

La Trinidad, Ocosingo, Tonalá, Arriaga, Ocozocuautla

Tuxtla Gutiérrez, El Chorreadero, Cinco Cerros, Berriozábal, Rizo de Oro, El Aguacero

Laguna Bélgica, Simojovel, Chicultic, Jitotol

Laguna Bélgica

No confirmado

El Triunfo

Villa Las Rosas

Laguna Bélgica, Berriozábal

Pacvilná, Tzunum

No confirmado

El Triunfo

Tapachula

Trinitaria, Laguna Bélgica, Simojovel, Cinco

Cerros, Chincultic

El Triunfo

Laguna Bélgica, Lago de Montebello, Chincultic, El Chorreadero, Simojovel, Yalumá.

Tapachula, Laguna Bélgica

San Cristóbal de Las Casas, Yalumá.

No confirmado

Chiapa de Corzo, Ocozocuautla, Cinco Cerros, Piedra Escrita, Villaflores, Villa Las Rosas, El Triunfo. 
Continúa

\begin{tabular}{|c|c|}
\hline ESPECIES & DISTRIBUCIÓN \\
\hline Phyllophaga aegrota (Bates, 1888) (2) (5) (23) & $\begin{array}{l}\text { Reserva El Ocote, Boca de Chajul, Laguna } \\
\text { Bélgica, El Chorreadero, Tzunum }\end{array}$ \\
\hline Phyllophaga anolaminata (Moser, 1921) (1) (18) & Villa Las Rosas \\
\hline Phyllophaga bucephala (Bates, 1888) (20) & El Triunfo \\
\hline Phyllophaga caanchaki Morón, 1998 (18) & Pico de Oro? \\
\hline Phyllophaga chamula Morón, 1999 (2) (15) (18) & $\begin{array}{l}\text { Teopisca, Navil, Tzunum, San Cristóbal de Las } \\
\text { Casas, }\end{array}$ \\
\hline Phyllophaga chiapas Saylor, 1943 (18) (20) (24) & El Triunfo \\
\hline Phyllophaga chiapensis (Chapin, 1935) (3) (18) & Yalumá, Aguacatenango \\
\hline Phyllophaga chimoxtila Morón, 2003 (17) (18) & El Bosque, Laguna Bélgica, Ocozocuautla \\
\hline Phyllophaga cholana Morón, 2003 (17) (18) & El Ocote, Laguna Bélgica, Ocozocuautla \\
\hline Phyllophaga cinnamomea (Blanchard, 1850) (5) (23) (24) & $\begin{array}{l}\text { (18) Reserva El Ocote, Laguna Bélgica, Boca de } \\
\text { Chajul }\end{array}$ \\
\hline Phyllophaga cometes (Bates, 1888) (1) (3) (5) (18) (23) (24) & $\begin{array}{l}\text { Reserva El Ocote, Boca de Chajul, Villa Las } \\
\text { Rosas, Aguacatenango, Majosik, Tenejapa, } \\
\text { Teopisca y Yalumá. }\end{array}$ \\
\hline Phyllophaga ciliatipes (Blanchard, 1850) (18) & Selva Negra \\
\hline Phyllophaga dasypoda (Bates, 1888) (18) (24) & Cintalapa \\
\hline \multicolumn{2}{|l|}{ Phyllophaga densata (Moser, 1918) (16) (18) } \\
\hline & $\begin{array}{l}\text { Ocosingo, Montes Azules, Ocozocuautla y } \\
\text { Laguna Bélgica }\end{array}$ \\
\hline Phyllophaga disca Saylor, 1943 (18) & Cintalapa \\
\hline $\begin{array}{l}\text { Phyllophaga enkerliniana Morón y Deloya, } \\
1998 \text { (4) (16) (18) (20) }\end{array}$ & El Triunfo municipio de Angel Albino Corzo \\
\hline Phyllophaga fulviventris Moser, 1918 (18) (24) & Ocozocuautla \\
\hline Phyllophaga guatemala Saylor, 1940 (2) (18) & Pacvilná, Tzunum \\
\hline Phyllophaga ginigra Saylor, 1940 (17) (18) & Palenque, Ocozocuautla, Ishuatlán \\
\hline Phyllophaga halffteriana Morón, 1992 (3) (18) (24) & Yalumá \\
\hline Phyllophaga heynei (Moser, 1918) (18) (24) & Huixtla \\
\hline Phyllophaga itsmica Morón, 2000 (18) & El Triunfo \\
\hline Phyllophaga integra Saylor, 1835 & Villa Las Rosas \\
\hline Phyllophaga integriceps (Moser, 1918) (18) & Cintalapa \\
\hline Phyllophaga javepacuana Morón, 2003 (17) (18) & $\begin{array}{l}\text { Trinitaria, Lagos de Montebello, Ocosingo, } \\
\text { Santa Rosa, Ocozocuautla, Laguna Bélgica }\end{array}$ \\
\hline Phyllophaga jovelana Morón y Cano, 2000 (18) (19) & $\begin{array}{l}\text { San Cristóbal de Las Casas, Lagos de } \\
\text { Montebello, Teopisca }\end{array}$ \\
\hline Phyllophaga latipes Bates, 1888 (18) (24) & Palenque \\
\hline Phyllophaga lenis (Horn, 1887) (1) (3) (24) & Villa Las Rosas, Yalumá \\
\hline Phyllophaga lineatoides Morón, 2000 (2) (3) (18) & Navil, Pacvilná, Tzunum, Piedra Escrita \\
\hline Phyllophaga longifoliata (Moser, 1921) (2) (3) & Pacvilná, Tzunum, Piedra Escrita \\
\hline Phyllophaga nandalumia Morón and Riley, 2005 (22) & Berriozabal, Ocozocuautla, \\
\hline
\end{tabular}


Pacheco Flores et al.: Fauna de escarabajos melolóntidos (Coleoptera: Scarabeoidea) en Chiapas, México

Continúa

\begin{tabular}{cl}
\hline ESPECIES & \multicolumn{1}{c}{ DISTRIBUCIÓN } \\
\hline Phyllophaga menetriesi (Blanchard, 1850)(1)(2)(3)(18)(24) & Pacvilná, Tzunum, Villa Las Rosas, \\
& Aguacatenango, Amatenango del Valle, El \\
& Madronal, Yalumá, San Francisco, Piedra \\
& Escrita, San Cristóbal de Las Casas, Yalumá, \\
& Aguacatenango \\
Phyllophaga multipora (Bates, 1888)(1)(18)(24) & Villa Las Rosas
\end{tabular}

Phyllophaga obsoleta (Blanchard, 1850) (1) (2) (3) (5) (9) (18) (20) (24)

Reserva El Ocote, San Cristóbal de Las Casas, Laguna Bélgica, Navil, Pacvilná, Tzunum, Villa Las Rosas, Aguacatenango, Amatenango del Valle, Balun Canal, Bochil, El Madronal, Juznajab, Majosik, Oxchuc, Piedra Escrita, Yalumá.

Phyllophaga ocozocuana Morón, 2003 (17) (18)

Ocozocuautla, El Ocote, Cuauhtémoc, El Bosque

Comalapa

Phyllophaga ome Morón, 1992 (18) (24)

Villa Las Rosas

Phyllophaga parvisetis (Bates, 1888) (1) (18) (24)

Phyllophaga pilula (Moser, 1921) (16) (18)

Phyllophaga pilosula (Moser, 1918) (2) (3) (9)

Phyllophaga pubicauda (Bates, 1888) (18) (24)

Phyllophaga pubicollis (Blanchard, 1850) (18) (24)

Phyllophaga punctuliceps (Bates, 1888) (18) (24)

Phyllophaga pruinosa (Blanchard, 1850) (6)

Phyllophaga ratcliffeiana Morón, 1992 (18) (24)

Phyllophaga ravida (Blanchard, 1850)(2) (3) (9) (18) (20) (24)

Phyllophaga rorulenta (Burmeister, 1855) (18)

Phyllophaga rostripyga (Bates, 1888) (24)

Phyllophaga rugipennis (Schauffus, 1858) (1) (2) (3) (16) (18) (24)

Phyllophaga rugulosa (Blanchard, 1851) (2) (3) (17) (18) (24)

San Cristóbal de Las Casas

Aguacatenango, Navil, Pacvilná, Piedra Escrita, Las Piedrecitas, San Cristóbal de Las Casas, Tenejapa, Tzunum, Winikton, Aguacatenango

Cinco Cerros

El Chorreadero

Montebello

Ocozocuautla

Ocozocuautla

Navil, Villa Las Rosas, Amatenango del Valle, El Madronal, Piedra Escrita, San Cristóbal de Las Casas, Teopisca, Yalumá, San Francisco, El Triunfo

Palenque

No confirmada

Ocosingo, Tapilula, La Trinidad, Laguna Bélgica, Huixtla, Pacvilná, Tzunum, Lago de Colores, San Cristóbal de Las Casas, Tenejapa, Majosik, Piedra Escrita, Palenque, Ocosingo, Ocozocuautla

Pacvilná, Ishuatlán, Tzunum, Pueblo Nuevo, Palenque, Lagos de Colores, Tzizcao, Oxchuc, Piedra Escrita, Tenejapa 


\begin{tabular}{|c|c|}
\hline ESPECIES & DISTRIBUCIÓN \\
\hline Phyllophaga rufotestacea (Moser, 1918) (3) (9) (25) & San Cristóbal de Las Casas \\
\hline Phyllophaga scabripyga (Bates, 1888) (2) (3) (18) & Tzunum, Yalumá, Aguacatenango \\
\hline Phyllophaga setidorsis (Bates, 1888) (18) (24) & Selva Negra \\
\hline Phyllophaga senicula Bates, 1888 (2) (3) (9) (18) (20) (24) & $\begin{array}{l}\text { Teopisca, Navil, Pacvilná, Tzunum, San } \\
\text { Cristóbal de Las Casas, Piedra Escrita, Las } \\
\text { Piedrecitas, Tenejapa, Tzunum y El Triunfo }\end{array}$ \\
\hline Phyllophaga soctona Morón, 2000 (1) (18) & Villa Las Rosas \\
\hline Phyllophaga sturmi (Bates, 1888) (5) (18) (24) (26) & Reserva El Ocote y Yaxchilán \\
\hline Phyllophaga stzotzilana Morón, 2001 (16) (18) & $\begin{array}{l}\text { Cañón del Sumidero, Mirador La Coyota, } \\
\text { Ocozocuautla, El Ocote, Ejido Nueva } \\
\text { Providencia }\end{array}$ \\
\hline Phyllophaga temora Saylor, 1943 (1) & Villa Las Rosas \\
\hline \multicolumn{2}{|l|}{ Phyllophaga tenuipilis (Bates, } \\
\hline 1888) (1) (3) (5) (16) (18) (23) (24) (26) & $\begin{array}{l}\text { Reserva El Ocote, Yaxchilán, Ocosingo, } \\
\text { Trinitaria, Tapilula, Boca de Chajul, Villa Las } \\
\text { Rosas, Albino Corzo, Finca Prusia, Berriozábal, }\end{array}$ \\
\hline & $\begin{array}{l}\text { Ocozocuautla, El Suspiro, Yalumá, } \\
\text { Aguacatenago, Teopisca, Amatenango del Valle, } \\
\text { El Madronal, Yalumá, Tuxtla Gutiérrez y San } \\
\text { Francisco }\end{array}$ \\
\hline \multicolumn{2}{|l|}{ Phyllophaga testaceipennis (Blanchard, } \\
\hline 1850$)(1)(2)(3)(9)(18)(23)(24)(26)$ & $\begin{array}{l}\text { Yaxchilán, Boca de Chajul, Navil, Pacvilná, } \\
\text { Tzunum, Aguacatenango, Amatenango del } \\
\text { Valle, El Madronal, Piedra Escrita, Yaluma, San } \\
\text { Francisco, Jusnajab, Majosik, Oxchuc }\end{array}$ \\
\hline Phyllophaga tojolabala Morón, 1999 (15) (18) & San Cristóbal de Las Casas \\
\hline Phyllophaga trichia (Bates, 1888) (24) & Tapilula \\
\hline \multicolumn{2}{|l|}{ Phyllophaga tumulosa (Bates, } \\
\hline 1888) (1) (2) (3) (9) (18) (24) (26) & $\begin{array}{l}\text { Yaxchilán, Ocozocuautla, San Cristóbal de Las } \\
\text { Casas, Navil, Villa Las Rosas, Aguacatenango, } \\
\text { Amatenango del Valle, Em Madronal, San } \\
\text { Francisco, Bochil, Navil, Tenejapa y Teopisca }\end{array}$ \\
\hline Phyllophaga vexata (Horn, 1885) (27) & El Chorreadero, Ocozocuautla \\
\hline Phyllophaga xanthocoma (Bates, 1888) (18) (24) & Laguna Bélgica \\
\hline Phyllophaga xkumuka Morón, 1999 (15) (18) & Trinitaria, Tuxtla Gutiérrez, Ocozocuautla \\
\hline Phyllophaga yaxbitana Morón, 2000 (18) & Rincón Chamula \\
\hline Phyllophaga wittkugeli (Nonfried, 1891) (1) & Villa Las Rosas \\
\hline Phyllophaga zunilensis (Bates, 1888) (18) (24) & Frontera Comalapa \\
\hline
\end{tabular}


Pacheco Flores et al.: Fauna de escarabajos melolóntidos (Coleoptera: Scarabeoidea) en Chiapas, México

Continúa

ESPECIES

DISTRIBUCIÓN

\section{Subfamilia Rutelinae}

Anomala ayjikcala Morón y Nogueira, 2002

Anomala atomograma Bates, 1888 (24)

Anomala bimaculata Blanchard, 1850 (23)

Anomala cincta Say, 1835 (2) (23) (24)

Anomala cupricollis Chevrolat, 1834 (26)

Anomala decolor Bates, 1888(1)

Anomala denticollis Bates, 1888 (1) (2) (3) (24)

Anomala discoidalis Bates, 1888 (1) (24)

Anomala donovani Stephens, 1830 (1) (24)

Anomala doryphorina Bates, 1888 (24)

Anomala eucoma Bates, 1888 (11) (23) (24)

Anomala forreri Bates, 1888 (1) (3)

Anomala flavilla Bates, 1888 (24)

Anomala flavizona Bates, 1888 (11) (24)

Anomala foraminosa Bates, 1888 (23) (24) (26)

Anomala granulipyga Bates, 1888 (15)

Anomala guatemalena Bates, 1888 (20) (24)

Anomala histrionella Bates, 1888 (11) (24)

Anomala hoepfneri Bates, 1888 (11) (24)

Anomala inconstans Burmeister, 1847 (1) (3) (9) (11) (24) (26)

Anomala laesicollis Bates, 1888 (24)

Anomala lepida Burmeister, 1844 (24)

Anomala marginicollis Bates, 1888 (23) (26)

Anomala megalops Bates, 1888 (11) (24)

Anomala micans Burmeister, 1847 (24)

Anomala millepora Bates, 1888 (24)
San Cristóbal de Las Casas, Teopisca

Montebello

Boca de Chajul

Boca de Chajul y Pacvilná

Yaxchilán

Villa Las Rosas

Pacvilná, Tzunum, Villa Las Rosas, Aguacatenango, El Madronal, Piedra Escrita, Teopisca, Winiktón y Yalumá

Villa Las Rosas

Villa Las Rosas

Tapilula

Huixtla, Tapachula, Laguna Bélgica y Boca de Chajul

Villa Las Rosas, Aguacatenango

El Chorreadero, Ocozocuautla

Laguna Bélgica

Yaxchilán, Boca de Chajul

No confirmada

El Triunfo

Reserva El Ocote, Laguna Bélgica, El Aguacero, Cinco Cerros, El Chorreadero, Huixtla, Laguna Bélgica, Comitán y Berriozábal

Laguna Bélgica, El Aguacero, Huixtla, Rizo de Oro, Ocozocuautla, Berriozabal

Ocozocuautla, Laguna Bélgica, Yaxchilán, El Aguacero, Chicoasén, Huixtla, El Chorreadero, Ocozocuautla, Berriozábal, Villa Las Rosas, Yalumá, Amatenango del Valle, Aguacatenango, El Madronal, San Cristóbal, Tenejapa y Teopisca.

No confirmada

No confirmada

Yaxchilán, Boca de Chajul, Laguna Bélgica

Berriozabal, Laguna Bélgica, Palenque, Simojovel

Tuxtla Gutiérrez, Ocozocuautla, La Trinitaria No confirmada 
Continúa

\begin{tabular}{l}
\hline ESPECIES \\
Anomala mixeana Morón y Nogueira, 2002 \\
Anomala nitidula Blanchard, 1850 (24) \\
Anomala ochroptera Bates, 1888 (2) \\
Anomala picturella Morón y Nogueira, 2002 (3) (9)(20)(2) \\
Anomala plurisulcata Bates, 1888 (24) \\
Anomala ruatana Bates, 1888 (24) (26) \\
Anomala specularis Bates, 1888 (24) \\
Anomala sticticoptera Blanchard, 1850 (1)(2)(3)(20)(24)
\end{tabular}

Anomala subaenea Nonfried, 1893 (24)

Anomala testaceipennis Blanchard, 1856 (24) DISTRIBUCIÓN

Tuxtla Gutiérrez, Cañón del Sumidero, Ocozocuautla, El Ocote, El Aguacero

Simojovel

Navil

Navil, Pacvilná, Tzunum, El Triunfo, San Cristóbal de Las Casas, Tenejapa

El Aguacero, Ocozocuautla, El Chorreadero, Berriozábal

Yaxchilán

No confirmada

Tuxtla Gutiérrez, Navil, Pacvilná, Tzunum, Villa Las Rosas, San Cristóbal de Las Casas, Piedra Escrita, Aguacatenango, Tenejapa, Balun Canal, Bochil, El Madronal, Teopisca, Yalumá y Winikton

Cinco Cerros, El Chorreadero, Chicoasén, Custepeques, Rizo de Oro, Totolapa, Comitán No confirmada

Anomala trapezifera Bates, 1888 (1) (2) (3) (9) (20)(23) (24) Boca de Chajul, Navil, Tzunum, Villa Las Rosas, Aguacatenango, San Cristóbal de Las Casas, Piedra Escrita, Tenejapa

Anomala undulata Melsheimer, 1844 (20) (24)

Tuxtla Gutiérrez, Jitotol, El Triunfo

Anomala valida Burmeister, 1844 (11) (24)

Laguna Bélgica, El Aguacero, Simojovel, Palenque, VillaFlores

Anomala vanpatteni Bates, 1888 (24)

No confirmada

Anomala villosella Blanchard, 1850 (24)

No confirmada

Anomala zapotensis Bates, 1888 (24)

Huixtla

Callistethus marginicollis (Bates, 1888) (5) (24)

Berriozábal, Reserva El Ocote

Callistethus vidua (Newman, 1841) (24)

Callistethus bimaculatus Blanchard, 1850 (24)

Tapachula, Cuxtepeques, Jitotol, Selva Negra

Boca de Chajul

Callistethus cupricollis Chevrolat, 1834 (5)

Reserva El Ocote, El Aguacero, Laguna Bélgica, Nonampak, Simojovel, Yaxchilán

Calomacraspis haroldi (Candeze, 1869) (24) Palenque

Calomacraspis splendens (Burmeister, 1844) (5) (11) (24) Reserva El Ocote, Laguna Bélgica, Ocuilapa,

Cnemida aterrima Bates, 1888 (11) (24)

Ocozocuautla, Chicoasén

Chasmodia collaris Blanchard, 1850 (5) (24)

Huixtla, Laguna Bélgica, Berriozábal, Cacahoatán, Palenque, El Aguacero

Najá, Boca de Chajul, Reserva El Ocote

Chlorota limbaticollis Blanchard, 1850 (5) (23) (24) (26)

Santa Rosa, Boca de Chajul, Yaxchilán, Reserva

El Ocote, Laguna Bélgica

Chrysina triumphalis Morón, 1990 (12) (20) (24)

El Triunfo, Albino Corzo, Lagos de Montebello 
Pacheco Flores et al.: Fauna de escarabajos melolóntidos (Coleoptera: Scarabeoidea) en Chiapas, México

Continúa

\begin{tabular}{|c|c|}
\hline ESPECIES & DISTRIBUCIÓN \\
\hline Epectinaspis bisyrica Paucar, 2002 & Cacaohatán, El Triunfo \\
\hline Heterosternus buprestoides Dupont, 1832 (24) & $\begin{array}{l}\text { Ocozocuautla, Berriozábal, } \\
\text { Montebello, Ocuilapa }\end{array}$ \\
\hline Heterosternus rodriguezi Candeze, 1869 (24) & $\begin{array}{l}\text { Tapachula, Lagos de Montebello, Tacana, } \\
\text { Cacahoatán y Motozintla }\end{array}$ \\
\hline Macraspis chrysis (Linné, 1764) (21) & Ocuilapa, La Flor \\
\hline Macraspis aterrima (Waterhouse, 1881) (21) (24) & $\begin{array}{l}\text { Monte Bello, Cinco Lagos, Cacaohatán, } \\
\text { Huixtla, Ocosingo, Chajul, El Aguacero, El } \\
\text { Sumidero }\end{array}$ \\
\hline Macraspis rufonitida Burmeister, 1844 (21) (23) (26) & Yaxchilán?, Monte Bello, Boca de Chajul?. \\
\hline Mesosternus halffteri Morón, 1987 (20) (24) & Oxchuc, Tapilula, El Triunfo, Albino Corzo \\
\hline Macropoides crassipes crassipes (Horn, 1866) (5) (11) (24) (26) & $\begin{array}{l}\text { Ocozocuautla, Ocosingo, Berriozábal, Reserva } \\
\text { El Ocote, Laguna Bélgica, Yaxchilán, El } \\
\text { Aguacero, Chicoasén, Cuilapa }\end{array}$ \\
\hline Macropoides cribicollis (Ohaus, 1934) (24) & $\begin{array}{l}\text { Tapilula, El Bosque, Huixtla, Tuxtla Chico, } \\
\text { Bochil, La Trinidad }\end{array}$ \\
\hline Macropoidelimus mniszechi (Sallé, 1873) (5) (11) (23) (24) (26) & $\begin{array}{l}\text { Berriozábal, Ocosingo, Ocozocuautla, Reserva } \\
\text { El Ocote, Laguna Bélgica, Yaxchilán, Boca de } \\
\text { Chajul }\end{array}$ \\
\hline Plesiorutela specularis (Bates, 1888 )(23) & Boca del Chajul, El Aguacero, Palenque \\
\hline Parisolea pallida (Candeze, 1869) (20) (24) & San Cristóbal de Las Casas, Chilil, El Triunfo \\
\hline Parisolea pachytarsis (Morón 1987) (20) (24) & El Triunfo, Albino Corzo \\
\hline Phalangogonia obesa Burmeister, 1844 (24) & Laguna Bélgica \\
\hline Phalangogonia ratcliffei Smith \& Morón, 2003 & Zanatepec, Rizo de Oro \\
\hline Plusiotis auropunctata Ohaus, 1913 (12) (24) & "Tapachula" probablemente hacia Tacaná \\
\hline Plusiotis chloreis Bates, 1888 (24) & Palenque, Malpaso \\
\hline Plusiotis diversa Ohaus, 1912 (12) (23) (24) & $\begin{array}{l}\text { Amatán, Palenque, Las Margaritas, Boca de } \\
\text { Chajul }\end{array}$ \\
\hline Plusiotis halffteri Morón, 1990 (24) & Oxchuc \\
\hline Plusiotis moroni Curoe \& Beraud, 1994 (20) (24) & $\begin{array}{l}\text { El Triunfo (Albino Corzo), Buenos Aires-El } \\
\text { Porvenir }\end{array}$ \\
\hline Plusiotis pehlkei Ohaus, 1930 (24) & Teopisca, Chilil \\
\hline Plusiotis psittacina Sturm, 1843 (12) (24) & $\begin{array}{l}\text { Finca Prusia, Albino Corzo, Agrónomos } \\
\text { Mexicanos }\end{array}$ \\
\hline Plusiotis quetzalcoatli Morón, 1990 (12) (20) (24) & $\begin{array}{l}\text { Bochil-Tapilula, Jitotol, La Hierbabuena, San } \\
\text { Cristóbal de Las Casas, Teopisca, Chilil, } \\
\text { Mitzitón, El Triunfo. }\end{array}$ \\
\hline Plusiotis quiche Morón, 1990 (20) & El Triunfo \\
\hline Plusiotis turckheimi Ohaus, 1913 (12) (24) & Hacienda San Pedro \\
\hline Plusiotis victorina Hope, 1840 (24) & Rizo de Oro? \\
\hline
\end{tabular}


Continúa

\begin{tabular}{|c|c|}
\hline ESPECIES & DISTRIBUCIÓN \\
\hline Pelidnota aurescens Bates, 1888 (11) (24) & $\begin{array}{l}\text { Laguna Bélgica, El Aguacero, Huixtla, } \\
\text { Tapachula, Cuxtepeques, VillaFlores, Cinco } \\
\text { Cerros }\end{array}$ \\
\hline Pelidnota centroamericana Ohaus 1913 (5) (11) (23) (24) (26) & $\begin{array}{l}\text { Reserva El Ocote, Laguna Bélgica, Yaxchilán, } \\
\text { Boca de Chajul, Palenque y Simojovel }\end{array}$ \\
\hline Pelidnota frommeri Hardy, 1975 (24) & Palenque \\
\hline Pelidnota guatemalensis Bates, 1888 (1) (3) (11) (24) & $\begin{array}{l}\text { Laguna Bélgica, E1 Sumidero, Comitán, } \\
\text { Teopisca, Aguacatenango, Yalumá }\end{array}$ \\
\hline Pelidnota notata Blanchard, 1850 (5) (11) (23) (24) (26) & $\begin{array}{l}\text { Reserva El Ocote, Laguna Bélgica, Yaxchilán, } \\
\text { Boca de Chajul, Malpaso, Palenque, Simojovel, } \\
\text { Bonampak, Solosuchiapa }\end{array}$ \\
\hline Pelidnota punctulata Bates, 1888 (5) (11) (24) (26) & $\begin{array}{l}\text { Reserva El Ocote, Laguna Bélgica, Yaxchilán, } \\
\text { El Aguacero, Cinco Cerros, Arriaga, Puerto } \\
\text { Arista, Simojovel, Tonalá, Tapachula, Palenque, } \\
\text { Ocozocuautla }\end{array}$ \\
\hline Pelidnota strigosa Laporte, 1840 (24) & El Aguacero, Tapachula, Ocozocuautla \\
\hline Pelidnota velutipes (Arrow, 1900) (24) (26) & Yaxchilán, Palenque \\
\hline Pelidnota virescens (Burmeister, 1844) (27) & El Aguacero, Cinco Cerros \\
\hline Platycoelia humeralis Bates, 1888 (27) & Tapilula, Lagos Montebello \\
\hline Leucothyreus femoratus Burmeister, 1844 (3) (23) (24) & Chajul, Yalumá \\
\hline Viridimicus aurescens (Bates, 1888) (3) (9) (20) (24) & $\begin{array}{l}\text { San Cristóbal de Las Casas, Chilil, Teopisca, El } \\
\text { Triunfo (Angel Albino Corzo) }\end{array}$ \\
\hline Strigoderma aterrima (Casey, 1915) (presente estudio) & Francisco Villa mpio. de Villaflores \\
\hline Strigoderma castor (Newman, 1841) (24) & No confirmada \\
\hline Strigoderma festiva Bates, 1888 (27) & Laguna Bélgica \\
\hline Strigoderma lampra Bates, 1888 (24) & No confirmada \\
\hline Strigoderma longicollis Bates, 1888 (24) & No confirmada \\
\hline Strigoderma mexicana Blanchard, 1850 (24) & $\begin{array}{l}\text { Laguna Bélgica, Lagos Montebello, Las } \\
\text { Margaritas }\end{array}$ \\
\hline Strigoderma orbicularis Burmeister, 1855 (24) & Palenque \\
\hline Strigoderma protea Burmeister, 1844 (27) & $\begin{array}{l}\text { La Sepultura, El Aguacero, Rizo de Oro, Cinco } \\
\text { Cerros }\end{array}$ \\
\hline Strigoderma vestita Burmeister, 1844 (5) & Reserva El Ocote, Jitotol \\
\hline Strigoderma sulcipennis Burmeister, 1844 (11) (24) & $\begin{array}{l}\text { Laguna Bélgica, El Aguacero, El Chorreadero, } \\
\text { Tuxtla Gutiérrez, Cinco Cerros, El Sumidero, La } \\
\text { Sepultura }\end{array}$ \\
\hline Strigoderma teapensis Bates, 1888 (27) & Palenque \\
\hline Strigoderma tomentosa Bates, 1888 (27) & Ocozocuautla \\
\hline Yaaxkumukia ephemera Morón y Nogueira, 2002 (10) & Jaltenango, El Triunfo, Tzizcao, Albino Corzo \\
\hline Yaakumukia conabioi Micó, Gómez and Galante, 2006 (10) & Unión Juárez, Cañada Talquian \\
\hline
\end{tabular}


Pacheco Flores et al.: Fauna de escarabajos melolóntidos (Coleoptera: Scarabeoidea) en Chiapas, México

Concluye

ESPECIES

DISTRIBUCIÓN

\section{Subfamilia: Trichiinae}

Apeltastes chiapensis Howden, 1994 (24) Cañón del Sumidero

Archedinus relictus Morón \& Krikken, 1990 (14) (20)(24) El Triunfo, A. Albino Corzo.

Giesbertiolus festivus (Howden, 1972) (24) Finca El Refugio

Inca clathrata sommeri Westwood, 1845 (5)(11) (24) La Trinitaria, Berriozábal, Tziscao, Simojovel,

Pantodinus klugi Burmeister, 1847 (24)

Paragnorimus sambucus Howden, 1970 (24)

Trigonopeltastes archimides Schaum, 1841 (27)

Trigonopeltastes frontalis Bates, 1889 (24)

Trigonopeltastes geometrica Schaum, 1841 (8)

Trigonopeltastes intermedia Bates, 1889 (24)

Trigonopeltastes s. sallei Bates, 1889 (11) (24)

Trigonopeltastes variabilis Howden, 1968 (24)

Trigonopeltastes glabella Howden, 1988 (24)

Reserva El Ocote, Laguna Bélgica

Unión Juárez, Volcán Tacaná, Boquerón

San Cristóbal de Las Casas

La Sepultura

Simojovel

Cintalapa

San Cristóbal de Las Casas, Chincultic

Comitán, Tuxtla Gutiérrez, Laguna Bélgica, El

Sumidero, El Aguacero, La Sepultura, Comitán

Simojovel

El Chorreadero, El Aguacero, El Sumidero

Trigonopeltastes thomasi H. \& R., 1990 (24)

El Sumidero

(1)Alcazar-Ruiz et al., 2003; (2)Castro-Ramírez et al., 2003; (3)Castro-Ramírez et al., 2005; (4)Deloya y Morón, 1998; (5)Gómez y Gómez, 1998; (6)Gómez y Gómez y Galdamez, 2001; (7)Gómez y Gómez y Chame, 2003; (8)Gómez y Gómez y Morales-Morales, 2003; (9)Méndez-Aguilar et al., 2005; (10)Micó et al., 2006; (11)Morales-Morales et al., 2005; (12)Morón, 1990; (13)Morón, 1995b; (14)Morón, 1995c; (15)Morón, 1999; (16)Morón, 2001; (17)Morón, 2003a; (18)Morón, 2003b; (19)Morón y Cano, 2000; (20)Morón-Ríos y Morón, 2001; (21)Morón y Paucar-Cabrera, 2003; (22)Morón and Riley, 2005; (23)Morón et al., 1985; (24)Morón et al., 1997; (25)Pacheco-Flores y Castro-Ramírez, 2005; (26)Palacios-Ríos et al., 1990; (27)Thomas, 1993.

Recibido: 6 de diciembre de 2006

Aceptado: 20 de septiembre de 2007 